Les ANNAles Les Annales de droit

DE DROIT

$8 \mid 2014$

Varia

\title{
Le (néo)normativisme, constructivisme inachevé
}

(Neo-)normativism, incomplete constructivism

\section{Marc Doray}

\section{OpenEdition}

Journals

Édition électronique

URL : https://journals.openedition.org/add/727

DOI : $10.4000 /$ add. 727

ISSN : 2606-1988

\section{Éditeur}

Presses universitaires de Rouen et du Havre

\section{Édition imprimée}

Date de publication : 1 mai 2014

Pagination : 145-196

ISBN : 979-10-240-0161-6

ISSN : 1955-0855

\section{Référence électronique}

Marc Doray, «Le (néo)normativisme, constructivisme inachevé », Les Annales de droit [En ligne], 8 |

2014, mis en ligne le 14 mars 2018, consulté le 08 décembre 2022. URL : http://

journals.openedition.org/add/727 ; DOI : https://doi.org/10.4000/add.727

Tous droits réservés 


\title{
Le (néo)normativisme, constructivisme inachevé
}

\author{
Marc DoraY
}

Le positivisme juridique n'est certes pas un courant d'analyse unifié et comporte de multiples variantes ${ }^{1}$, à tel point que l'emploi du singulier pour le caractériser pourrait passer pour un signe d'ignorance ou de

1. Uberto Scarpelli, déjà en 1965, pouvait donner trois représentations différentes $\mathrm{du}$ juspositivisme distinguant chacune au sein du positivisme, entre un mode d'approche, une théorie ou une idéologie: selon Norberto Bobbio, on peut ainsi distinguer le positivisme comme 1. Mode d'approche du droit caractérisé par une distinction nette entre le droit tel qu'il est et le droit tel qu'il devrait être, et par la conviction que le seul qui doive être étudié soit le premier, selon le principe de la Wertfreiheit (neutralité axiologique). 2. Une théorie stato-légale du droit ayant pour conséquence: $a$. une définition volontariste du droit; b. une conception impérative de la norme juridique; $c$. une théorie des sources du droit qui donne la prééminence à la loi; d. l'admission du caractère complet et cohérent de l'ordre juridique; e. une théorie d'après laquelle le rôle du juriste et du juge consiste principalement à dire le droit existant et non à produire des règles nouvelles. 3. Une idéologie selon laquelle le droit en vigueur doit être obéi, soit parce qu'il est considéré comme juste, soit parce qu'il permet la réalisation de la paix et l'ordre (Voir Norberto Bobbio, "Le positivisme juridique", dans Essais de théorie du droit, trad. Michel Guéret et Christophe Agostini, préface de Riccardo Guastini, Paris, LGDJ, «La pensée juridique », juin 2000, p. 23-27). Hart, lui, identifie cinq éléments indépendants les uns des autres: 1 . le droit est constitué de commandements issus d'êtres humains; 2. il n'y a pas de rapports nécessaires entre le droit et la morale ou entre le droit tel qu'il est et le droit tel qu'il devrait être; 3. l'analyse, ou l'étude de la signification des concepts juridiques, mérite d'être faite et se distingue à la fois des recherches sur les causes et les origines du droit, des enquêtes sociologiques sur les rapports du droit et des autres phénomènes sociaux, et de l'appréciation du droit en termes moraux ou en termes de buts sociaux, etc.; 4. un système juridique est un système logique fermé dans lequel des décisions correctes peuvent être logiquement déduites des normes qui préexistent sans qu'il soit fait référence à des buts sociaux, des intentions politiques ou des buts moraux; 5. les jugements moraux ne peuvent être établis ou défendus rationnellement comme les jugements de fait. On pourrait encore citer les conceptions positivistes telles que représentées par Mario Cattaneo (Voir Mario Cattaneo, Il positivismo giuridico inglese. Hobbes Bentham, Austin, Milan, Giuffré, 1962, chap. I, cité par Uberto Scarpelli, Qu'est-ce que le positivisme juridique, Paris, LGDJ, «La pensée juridique », novembre 1998, p. 14), celles d'Alf Ross (voir, pour un résumé, Michel Troper, «Positivisme», dans André-Jean Arnaud, Dictionnaire 
confusion ${ }^{2}$. C'est toutefois une dimension spécifique du positivisme juridique que nous nous proposons de mettre en avant ici : sa filiation avec le positivisme philosophique en tant que modalité de la connaissance. Il paraît par exemple possible à Michel Troper d'admettre l'assertion selon laquelle certains juristes «seraient positivistes non pas en raison d'un rattachement quelconque au courant positiviste en philosophie, mais uniquement parce qu'ils entendent décrire le droit "positif". [...] [Toutefois] le positivisme est [plutôt] une simple application au droit du positivisme philosophique et d'ailleurs la plupart des juspositivistes contemporains font de fréquentes références aux travaux des philosophes positivistes, notamment au courant de l'empirisme logique ${ }^{3}$ ». Le "positivisme » scientifique, en tant que doctrine de la connaissance, postule l'existence de l'objet d'étude "comme un fait, extérieur à son examinateur qui tente de le décrire ou de l'expliquer objectivement, à partir d'une observation ${ }^{4}$ ».

Ainsi, pour Raymond Carré de Malberg déjà, le chercheur en sciences juridiques (ou «juriste») «ne participe point, comme tel, à la tâche de la création du droit [et] n'a point, pour sa part, à pourvoir à la lex ferenda : il opère seulement au service de la lex lata. Il ne lui appartient pas de refaire

encyclopédique de théorie et de sociologie du droit [ci-après DETSD], Paris, LGDJ, "Droit et Société », 1993 ( $2^{\mathrm{e}}$ éd.), p. 461).

2. Selon Michel Troper, «En réalité, la plupart des définitions sur lesquelles se fondent ceux qui écrivent sur le positivisme [juridique] sont des définitions stipulatives", c'est-à-dire purement conventionnelles, entre les usagers futurs de l'expression (Michel Troper, Pour une théorie juridique de l'État, Paris, PUF, 1994, p. 29). Ainsi, pour Uberto Scarpelli, «Le choix et la pertinence d'une définition stipulative dépendront de l'utilité et de la signification qu'elle permet de déterminer, de la correspondance d'une telle signification aux fonctions et aux exigences du corpus linguistique dans lequel elle va être introduite» (Uberto Scarpelli, Qu'est-ce que le positivisme juridique, trad. Colette Clavreul, préface Laetizia Gianformaggio, ( ${ }^{\mathrm{re}}$ éd. 1965), Paris, LGDJ, « La pensée juridique », novembre 1996, p. 5-6).

3. Michel Troper, "Positivisme", op. cit., p. 462-463. Il est possible d'affiner cette lecture: le positivisme a largement inspiré les juristes à travers deux variantes. Le positivisme naturaliste de Comte, affirmé dans son Cours de philosophie positive (1830-1842 ; ouvrage fondateur du positivisme scientifique), présuppose que les valeurs (morales, politiques et, en ce qui nous intéresse, juridiques), sont connaissables. Le positivisme juridique a pris en compte la pensée d'un autre père fondateur du positivisme, David Hume, et intégré le non-cognitivisme éthique: on ne peut inférer, à partir de prémisses entièrement descriptives, de conclusions normatives. On retrouve ici la distinction Sein/Sollen reprise par Kelsen, « véritable pierre d'angle du positivisme non comtien appliqué à l'étude des phénomènes juridiques » (Éric Maulin, "Positivisme", dans Denis Alland et Stéphane Rials, Dictionnaire de la culture juridique, Paris, PUF, «Quadrige. Dicos Poche», août 2003, p. 1173).

4. Éric Maulin, «Positivisme», art. cité, p. 1171. 
le monde juridique, mais seulement de constater comme ce monde est fait ${ }^{5}$ ». Comme l'observe Éric Maulin: «cette attitude [...] aujourd'hui partagée par la plupart des auteurs qui se réclament du positivisme [...] implique, comme le soutiennent Hans Kelsen et H. L. A. Hart, que le scientifique se tienne dans un rapport d'extériorité par rapport à son objet $^{6} \ldots »$ De fait, Kelsen ne donne pas simplement une définition du droit mais également de la science juridique et de sa méthode. Dès lors: "La science du droit trouve son objet préconstitué, car le droit seul décide de ce qui est du droit (ou même, dans la version plus forte, seul le droit crée du droit). [Telle est] la position de Kelsen dans certains de ses écrits ${ }^{7} . . . »$ Pourtant, à l'étude, le positivisme juridique semble davantage correspondre à la définition du "constructivisme». Abordé notamment par Gaston Bachelard dès $1934^{8}$, puis par Jean Piaget $^{9}$, le constructivisme peut se définir comme un "discours sur les fondements de la connaissance scientifique ${ }^{10}$ » qui, « au postulat de la réalité [...], oppose [...] le principe de la représentabilité de nos expériences. [...] La connaissance n'est alors plus celle d'un objet et de son objectivité, comme elle l'était pour les épistémologies positivistes. Elle devient, dans une perspective d'interaction entre l'objet et le sujet, connaissance d'un projet, ce qu'on nomme principe de projectivité ${ }^{11}$ ". Plus simplement, l'objet d'étude n'apparaît plus comme un donné mais

5. Raymond Carré de Malberg, "Réflexions très simples sur l'objet de la science juridique", dans Recueil d'études sur les sources du droit en l'honneur de François Gény, Paris, Librairie du Recueil Sirey, 1937, t. I, p. 201 et suiv.

6. Éric Maulin, «Positivisme», art. cité, p. 1174.

7. Christophe Grzegorczyk, «Le positivisme comme méthodologie juridique. Introduction ", dans Christophe Grzegorczyk, Françoise Michaut et Michel Troper (dir.), Le Positivisme juridique, Paris, LGDJ, "La Pensée juridique moderne », octobre 1998, p. 191.

8. "Pour un esprit scientifique, toute connaissance est une réponse à une question. S'il n'y a pas eu de question, il ne peut y avoir connaissance scientifique. Rien ne va de soi. Rien n'est donné. Tout est construit " (Gaston Bachelard, La Formation de l'esprit scientifique. Contribution à une psychanalyse de la connaissance objective, $5^{\mathrm{e}}$ éd., Paris, J. Vrin, 1967, p. 14).

9. Le constructivisme de Jean Piaget s'est construit pendant plus de trente ans, de La construction du réel chez l'enfant (Jean Piaget, Paris, Delachaux et Niestlé, 1937) à Logique et connaissance scientifique (Jean Piaget (dir.), Paris, Encyclopédie de la Pléiade, $\mathrm{n}^{\circ}$ 22, mars 1967).

10. Jean-Louis Le Moigne, Le Constructivisme. Tome 1: Les enracinements, Paris, L'Harmattan, «Ingenium », juillet 2012, p. 13.

11. André-Jean Arnaud, «Epistémologie. 8 », dans André-Jean Arnaud, op. cit., p. 232. 
comme un construit, produit de l'interaction entre le sujet connaissant et l'objet de connaissance ${ }^{12}$.

Ce paradoxe du constat d'un positivisme (juridique) constructiviste, que nous nous proposons de démontrer, se double d'un aspect plus critique : le positivisme apparaît, non seulement comme un constructivisme mais, surtout, comme un constructivisme réducteur. Nous nous intéresserons ici aux thèses normativistes et néo-normativistes, dont Kelsen fut - et demeure - l'inspirateur, qui appartiennent toutes deux "au genre "positivisme ${ }^{13}$ " ". À l'encontre des thèses volontaristes dominantes au début $\mathrm{du} \mathrm{xx}^{\mathrm{e}}$ siècle, selon lesquelles le droit est le fruit d'un acte

12. Tel est le cœur de la l'épistémologie constructiviste, invariant malgré les multiples variantes de cette dernière, du constructivisme radical de prôné par Ernst von Glaserfeld («Introduction à un constructivisme radical», dans Paul Watzlawick (dir.), L'Invention de la réalité. Contributions au constructivisme, Paris, Seuil, "Points Essais», mai 1996, p. 19-43) au constructivisme «dialectique» de Jean Piaget (Logique et connaissance scientifique, op. cit., note 9), en passant par le constructivisme d'Edgar Morin, représentation de la complexité (voir notamment Edgar Morin, La Méthode, ( $1^{\text {re }}$ éd., mai 1986), Paris, Seuil, « Points Essais », t. III, «La connaissance de la connaissance», janvier 1992, 243 p.; Introduction à la pensée complexe, ( ${ }^{\text {re }}$ éd., ESF, 1990), Paris, Seuil, «Points Essais», avril 2005, 158 p.), au constructivisme comme modélisation des systèmes complexes (Voir Jean-Louis Le Moigne, La Modélisation des systèmes complexes, (1 ${ }^{\mathrm{re}}$ éd., 1990), Paris, Dunod, "Sciences humaines", février 2011, 178 p.) Pour une approche plus renseignée sur la multiplicité des auteurs ayant contribué, d'une manière ou d'une autre, à la pensée constructiviste, consulter Jean-Louis Le Moigne, Le Constructivisme, op. cit., p. 267-283).

13. Michel Troper, «Normativisme», art. cité, p. 396. On prendra soin ici de donner une définition explicative du "normativisme». Si deux sens apparaissent pouvoir être dégagés de l'expression «normativisme» («Terme par lequel certains commentateurs désignent la théorie du droit de Hans Kelsen »; «théorie appartenant au genre "positivisme", et qui envisage le droit comme un système de normes »), il nous paraît préférable d'accorder à la première de ces définitions, plus stricte, une primauté (chrono)logique. Les normativismes de Herbert Hart et Norberto Bobbio envisagent effectivement le droit comme un système de normes, mais font état de différences notables avec le normativisme de l'école de Vienne dont Kelsen reste le fondateur. Herbert Hart distingue ainsi un point de vue interne (celui adopté par Kelsen) et un point de vue externe (celui adopté par un sociologue, par exemple) et souligne qu'il est possible, et même souhaitable, d'envisager le droit selon un «point de vue externe modéré ou point de vue de l'observateur externe qui se réfère au point de vue interne des juristes » (François Ost, "Science du droit », dans André-Jean Arnaud, op. cit., p. 541). Quant à Norberto Bobbio, d'une part, il considère la science juridique non pas comme une «énigmatique science "normative", mais plus simplement [comme] une analyse linguistique : l'analyse du discours du législateur. La science du droit est ainsi réduite par Bobbio à un "discours sur le discours", un méta-langage descriptif, dont le langage-objet est le discours prescriptif du législateur » (Riccardo Guastini, «Norberto Bobbio ou de la distinction », dans 
de volonté, Kelsen propose à partir de 1911 une grille de lecture du droit totalement renouvelée que l'on peut résumer schématiquement comme suit : ce n'est pas parce qu'une certaine chose est voulue par l'État (ou ses représentants) qu'elle doit lui-être imputée, mais c'est parce que cette chose est imputée à l'État que l'on peut estimer que ce dernier l'a voulu. Dit autrement, ce n'est pas parce qu'il y a acte de volonté qu'il y a droit, mais l'inverse. Kelsen appuie sa thèse sur un raisonnement analytique qui recèle une double fragilité: il considère les normes comme «données » alors même qu'il adopte une position constructiviste, et forge pour ce faire la catégorie de l'imputation; il ne parvient pas à rendre compte de la validité du droit, en recourant à la structure conceptuelle unitaire qu'il a pourtant bâtie. Le caractère inachevé de la théorie kelsénienne trouve un écho dans les thèses de ses épigones, néo-normativistes, qui, malgré les différentes complications ou simplifications apportées au construit kelsénien, ne parviennent pas à s'affranchir des apories de celui-ci. Ainsi, normativisme et néo-normativisme apparaissent comme deux constructivismes imparfaits (1). Cet inaboutissement, toutefois, n'est pas le produit de l'épistémologie constructiviste adoptée (du moins, pas directement), mais résulte plutôt de la manière dont celle-ci a été travaillée par un projet commun et réducteur: la construction d'une science "pure», dont l'objet serait unique (2).

\section{Normativisme et néo-normativisme: deux constructivismes imparfaits}

Le normativisme kelsénien est aujourd'hui largement considéré comme un constructivisme inachevé (1.1), un caractère imparfait que les émules de Kelsen, qualifiés de néo-normativistes, n'ont pu, malgré leurs efforts, améliorer (1.2).

Norberto Bobbio, op. cit., p. 9). D’autre part, cette définition du droit comme langage du législateur amène le juriste (et philosophe) italien «à représenter le droit à la fois comme un fait et comme une norme» (ibid.), réconciliant ainsi deux théories en apparence antithétiques : le normativisme de Kelsen et le réalisme scandinave. Enfin, la généralisation consistant à faire du normativisme une théorie envisageant le droit comme un système de normes est une source incontestable de confusions puisque, tandis que Kelsen envisage le droit comme un système préconstitué de normes émanant uniquement de l'ordre juridique lui-même, Norberto Bobbio affirme au contraire que « le système juridique n'est en fin de compte pas du tout l'ensemble des normes expresses, énoncées par le législateur [ou les autres organes créateurs de droit mais] le résultat des opérations d'addition et de soustraction accomplies par les juristes sur l'ensemble des normes expresses formellement valides », ajoutant ainsi à la dimension formelle du concept de validité, une dimension matérielle (ibid., p. 11). 


\subsection{Le normativisme kelsénien, constructivisme inachevé ${ }^{14}$}

Le caractère inachevé du constructivisme de Kelsen apparaît dans le caractère inabouti de sa démonstration relative tant à la catégorie de l'imputation qu'à l'existence même de la norme comme «donné » (1.1.1); il se retrouve également dans l'échec de l'idée d'inférence, idée devant normalement permettre d'identifier le droit, c'est-à-dire le droit valide (1.1.2). Reste alors à déterminer les conséquences à tirer de ce constat (1.1.3).

\subsubsection{Norme et imputation chez Kelsen: deux constructions fragiles}

Il est possible de dresser une typologie des écrits de Kelsen (en fonction de leur appartenance à trois périodes distinctes de la vie du juriste autrichien, marquées par des logiques différentes voire, en ce qui concerne la période postérieure à 1961, contradictoires avec l'ensemble de son œuvre précédente): ainsi, Stanley Paulson opère une distinction entre la période "primitive" ou - et c'est à noter " constructiviste " (1911-1920 environ), la période "intermédiaire " (1920-1961) et la période «tardive» ou "volontariste». Nous nous intéresserons ici seulement aux deux premières en ce qu'elles révèlent les deux constructivismes essentiels de la pensée du juriste viennois ${ }^{15}$.

14. Il nous paraît toutefois important de souligner l'apport de Kelsen en nous associant aux propos de son plus grand adversaire, Alf Ross : «Une des qualités d'un grand travail scientifique ou philosophique est son pouvoir de stimulation. Si vous vous plongez dans ce travail, vous en tirerez toujours profit. Même si vous en venez à ne pas être d'accord, vous vous trouvez être également, d'une certaine façon, redevable à l'auteur du travail des perspectives qui s'offrent à vous lorsque vous confrontez vos idées aux siennes. Vous comprenez que, pour moitié, ces perspectives sont son œuvre, parce qu'elles résultent d'un processus dialectique qu'il a provoqué; et vous vous rappelez que si le moineau s'élève plus haut dans le ciel, c'est qu'il est sur le dos de l'aigle ( (Alf Ross, "Qu'est-ce que la justice selon Kelsen », dans Introduction à l'empirisme juridique, trad. Éric Millard et Elsa Matzner, Paris, LGDJ, 2004, p. 125).

15. Dans sa Théorie générale des normes (Paris, PUF, "Léviathan", 2011, ci-après $T G N$ ) Kelsen adhère en effet à une ontologie expressive des normes (la norme est la signification d'un acte de volonté, voir chap. I, $\$ 1$ à IV; chap. IX, $\$ 1$ à III ; chap. XXXIX ; chap. LVII) là où il faisait auparavant prévaloir une conception hylétique en distinguant la signification subjective - c'est-à-dire l'acte de volonté -, et la signification objective - c'est-à-dire l'attribution de la validité à cet acte de volonté par une autre norme -, seule capable de faire de cet acte de volonté une norme (voir notamment Hans Kelsen, Théorie pure du droit (ci-après TPD), $2^{\mathrm{e}}$ éd. ( $1^{\text {re }}$ éd. française de la $2^{\mathrm{e}}$ éd. allemande, Paris, Dalloz, 1962), trad. Charles Eisenmann, Bruxelles, Paris, LGDJ, «La pensée juridique », novembre 2010, p. 16. 
En 1911, dans sa thèse d'habilitation intitulée Hauptprobleme der Staatrechtslehre ${ }^{16}$, Hans Kelsen s'oppose au juspositivisme traditionnel de l'école Gerber-Laband-Jellinek qui assimilait la volonté de l'État à un concept psychologique "selon lequel un acte juridique est valide si [ou] parce qu'il est voulu psychologiquement ${ }^{17}$ ». Il opère en effet un renversement majeur en soutenant que l'acte juridique est voulu parce qu'il est considéré comme valide. Dès lors, la volonté de l'État n'apparaît plus comme une notion (psychique ou psychologique) donnée mais bien " comme une notion construite ${ }^{18}$ » : si une chose est imputée à l'État, alors on peut dire qu'elle est voulue par celui-ci. C'est dans sa Théorie pure $d u$ droit que Kelsen appuiera ce renversement constructiviste sur une autre construction: la célèbre "norme fondamentale" (Grundnorm). Il procède, pour ce faire, en deux temps ${ }^{19}$ :

- Il introduit d'abord le concept d' « imputation normative» comme catégorie fondamentale sur le modèle du principe de causalité : «Finalement, pour donner un schéma général et abstrait [de l'imputation] : dans telles et telles conditions, que détermine l'ordre juridique, un acte de contrainte qu'il définit doit avoir lieu. Les propositions juridiques lient donc l'un à l'autre deux éléments, exactement comme les lois naturelles. Mais voici maintenant la différence considérable: le lien qu'énonce la proposition juridique a une signification radicalement différente de celui

Parallèlement, dans la TGN, Kelsen affirme que la validité de la norme juridique "dépend de l'acte de volonté dont elle est la signification " et même que «la possibilité de parvenir à la validité de la norme individuelle par la voie d'une inférence logique à partir de la validité de la norme générale, ne peut, au fond, nullement être envisagée» (TGN, op. cit., chap. LviII, $\$ 10$, p. 314-315). Pour une même interprétation de l'abandon par Kelsen de ces deux thèses, voir Michel Troper, Pour une théorie juridique de l'État, op. cit., p. 59-63; Stanley Paulson, "Introduction", dans Hans Kelsen, Théorie générale du droit et de l'État (ci-après $T G D E$ ), suivi de La Doctrine du droit naturel et le positivisme juridique, trad. Valérie Faure, Paris, LGDJ, «La pensée juridique », décembre 2010, p. 7. Le plus important, toutefois, est que cet abandon ne saurait constituer, per se, une critique valable ou suffisante des thèses précédentes. Reste que le fait que Kelsen, après 1960, ait revu bon nombre de points de sa théorie ne saurait constituer, per se, une critique valable ou suffisante de ses thèses précédentes.

16. Hans Kelsen, Hauptprobleme der Staatrechtslehre entwickelt aus der Lehre vom Rechtssatze, Tübingen, J. C. B. Mohr, 1911.

17. Stanley Paulson, art. cité, p. 16.

18. Ibid. Voir également Stanley Paulson, "Hans Kelsen's Earliest Legal Theory: Critical Constructivism », The Modern Law Review Limited, 59 :6, novembre 1996, p. 797-812. L'auteur s'étonne de ce que le constructivisme de Kelsen n'ait jusqu'alors fait l'objet que de peu d'attention

19. On retrouve le développement de cet argument transcendantal néokantien dans Hans Kelsen, TPD, op. cit., p. 201-205. 
[de] la causalité. [...] Dans la proposition juridique, il n'est pas dit, comme dans la loi naturelle, que si A est, B est; il y est dit que, si A est (sein), B doit être (soll sein); et ceci n'implique nullement que B sera réellement chaque fois que $A$ sera. Le fait que la signification de connexion des deux éléments dans la proposition juridique ne soit pas identique à ce qu'elle est dans la loi naturelle a sa source dans cette donnée que, dans la proposition juridique, la connexion est établie par une norme posée par l'autorité juridique, c'est-à-dire par un acte de volonté, alors que la connexion de la cause à l'effet [...] énoncée dans la loi naturelle et indépendante de toute semblable intervention ${ }^{20}$."

- Puis, utilisant un argument transcendantal néo-kantien, implicite dans la norme fondamentale qu'il suppose ${ }^{21}$, il tente de démontrer que cette catégorie fondamentale de l'imputation est nécessairement présupposée par des éléments qui sont donnés, à travers un schéma analytique que l'on peut représenter, à l'instar de S. Paulson, comme suit :

1. Les normes juridiques sont connues (données);

2. La connaissance des normes juridiques n'est possible que si la catégorie de l'imputation normative (prémisse transcendantale) est présupposée;

3. Donc la catégorie de l'imputation normative est présupposée (conclusion transcendantale) ${ }^{22}$.»

Le schéma analytique de Kelsen présenté ci-dessus, est repris du raisonnement analytique kantien. Il s'en distingue toutefois par son caractère tronqué. En effet, le raisonnement analytique chez Kant est plus complexe que la version néo-kantienne reprise par Kelsen. Si, tout comme Kelsen, Kant affirme que "l'on part de ce que l'on cherche comme s'il était donné et [...] l'on remonte aux seules conditions sous

20. Ibid., chap. XVIII, p. 85 .

21. «En décrivant la norme fondamentale, nous n'introduisons aucune méthode nouvelle dans la science du droit. Nous ne faisons que rendre explicites ce que tous les juristes supposent, inconsciemment dans la plupart des cas, dès lors qu'ils considèrent le droit positif comme un système de normes valides et non comme un ensemble de faits, et qu'ils rejettent en même temps un droit naturel qui conférerait sa validité au droit positif. L'existence de la norme fondamentale dans la conscience juridique résulte d'une simple analyse des énoncés juridiques effectifs. La norme fondamentale répond à la question [transcendantale] : comment, et dans quelles conditions, les énoncés de droit relatifs aux normes juridiques, aux obligations juridiques, aux droits subjectifs, etc., sont-ils tous possibles? » (Hans Kelsen, TGDE, op. cit., p. 170).

22. Stanley Paulson, art. cité, p. 22. 
lesquelles il est possible ${ }^{23}$ ", il procède néanmoins à un dédoublement de ce raisonnement. D'abord une version dite "progressive", qui part d'éléments connus comme étant donnés; puis la version dite "régressive ", celle utilisée isolément par les néokantiens, dont Kelsen.

Ce que l'on peut représenter grâce au schéma analytique suivant ${ }^{24}$ :

\section{Version progressive}

1. $\mathbf{P}$ [phénomènes de conscience, en tant que données]

2. $\mathrm{P}^{\star} \mathrm{Q}$ [où « $\mathrm{Q}$ » représente la catégorie (de causalité) comme condition de $\mathrm{P}$, et ${ }^{*}{ }^{\star}$ » signifie « est seulement possible $\mathrm{si}^{25}$ »]

3. Q [catégorie (de causalité) comme conclusion : déduit de 2]

4. $\mathbf{R}$ [énoncé de connaissances, en tant que conclusion : déduit de 3] Puis

\section{Version régressive}

1. $\mathbf{R}$ [énoncé de connaissances, en tant que données]

2. $\mathbf{R}^{\star} \mathrm{Q}$ [catégorie comme condition]

3. Q [catégorie comme conclusion]

Alors que Kant, avec sa version progressive, part d'une prémisse modérée, rapportant des «données situées en-deçà du seuil de l'expérience interprétée ${ }^{26}$ », les néo-kantiens - dont Kelsen - n’utilisent que la version régressive du raisonnement analytique et partent donc d'une prémisse non démontrée par la version progressive. Au prix de la fragilité de tout le raisonnement. Ainsi, Kelsen part d'une donnée déjà interprétée (la norme), ce à quoi le sceptique ne peut adhérer et, en outre, sa seconde prémisse paraît trop exigeante, faisant de l'imputation le point obligé de la défense d'une théorie normativiste du droit.

Conscient de cette fragilité, Kelsen lui-même admet en 1960 que ce qu'il considère comme donné n'est en réalité qu'un construit, auquel on doit adhérer pour pouvoir appréhender le droit dans une perspective normativiste :

On [...] interprétera objectivement les relations entre personnes comme des relations de droit et, plus spécifiquement, comme des obligations

23. Emmanuel Kant, Prolégomènes à toute métaphysique future, trad. Louis Guillermit, cité par Stanley Paulson, art. cité, p. 24.

24. Schéma donné par Stanley Paulson, ibid., p. 22-26.

25. Le signe $" * *$ représente le caractère modal.

26. Stanley Paulson, art. cité, p. 28. 
juridiques, des droits subjectifs et des pouvoirs juridiques si et seulement si on présuppose la norme fondamentale. Ce n'est là toutefois qu'une interprétation possible, une interprétation rendue possible par le présupposé de la norme fondamentale et dépendant de ce présupposé; ce n'est pas une interprétation nécessaire. Les relations entre personnes peuvent également être interprétées comme de simples relations de pouvoir, c'est-à-dire comme des causes et des effets, conformément à la loi de causalité ${ }^{27}$.

Ce premier point marque le caractère inachevé du constructivisme kélsénien. Le réaliste danois Alf Ross ne manquera d'ailleurs pas de souligner cette fragilité : si la norme fondamentale de Kelsen n'est qu'un présupposé, extrajuridique, alors il n'y a guère de différence entre le positivisme de Kelsen et une forme quelconque de jusnaturalisme ${ }^{28}$. L'interaction de l'observateur scientifique avec son objet ne fait, pour A. Ross, aucun doute. Pourtant, bien que cette critique ait portée, elle ne semble pas dirimante au regard de la Théorie pure du droit revendiquée par H. Kelsen ${ }^{29}$.

27. Hans Kelsen, «On the Basis of Legal Validity» (1960), traduit par Stanley Paulson, American Journal of Jurisprudence, $\mathrm{n}^{\circ}$ 26, 1981, p. 178-189 et 185-186.

28. «... La validité, au sens normatif, n'a pas de fonction descriptive ou explicative de la réalité. Sa fonction est de renforcer l'ordre juridique en proclamant que les obligations prescrites par cet ordre juridique [...] constituent aussi des devoirs moraux. La notion normative de validité est l'instrument d'une idéologie qui soutient l'autorité de l'État. [...] Bien que cette implication de la pensée de Kelsen ne s'accorde pas avec le programme empiriste proclamé par la théorie pure du droit, elle est inévitable et doit être comprise comme la survivance d'une philosophie jusnaturaliste de l'espèce quasi-positiviste. [...] Il faut noter à cet égard que le présupposé, selon lequel la norme fondamentale confère à l'ordre effectif la validité, est attribué à ce que Kelsen appelle la "pensée juridique". Ce présupposé n'est révélé - et accepté - que par la science du droit. Par "pensée juridique", on vise, je suppose, les idées et croyances généralement acceptées par les juristes. Mais la "pensée juridique" n'est pas un instrument fiable pour une analyse logique. Il se peut [...] que la manière habituelle de penser soit saturée de concepts idéologiques, correspondant à des expériences affectives, mais tout à fait inadéquats pour décrire la réalité, ce qui est pourtant la tâche de la science du droit » (Alf Ross, "Validity and the conflict between Legal Positivism and Natural Law», Revista Juridica de Buenos Aires, IV, 1961, fragments des p. 72, 74, 78, cité et traduit par Christophe Grzegorczyk dans Le Positivisme juridique, op. cit., p. 204-205).

29. Tout appareil conceptuel scientifique nécessite une loi fondamentale, érigeant les critères d'appréciation de la scientificité des propositions décrivant les phénomènes objets de l'étude. Or, cette loi ne peut elle-même correspondre aux critères qu'elle pose. Elle est donc nécessairement extérieure au système qu'elle instaure et repose sur un présupposé communément admis au sein de la communauté scientifique concernée. La contrôlabilité empirique des propositions correspond à la loi fondamentale des sciences purement causales, telles les sciences physiques. Comme le soulignent Kelsen lui-même et Andràs Jakab à sa suite, la norme fondamentale 


\subsubsection{Une validité formelle non démontrée}

L'inachèvement lié au caractère tronqué de l'argument transcendantal kelsénien se double du non-respect par Kelsen lui-même de la distinction, pourtant au cœur de la catégorie fondamentale de l'imputation, entre l'être (Sein) et le devoir-être (Sollen). Le principe de cette distinction réside dans ce que l'on appelle parfois «Loi de Hume» dont la teneur peut être résumée comme suit :

Personne ne peut nier que, du fait que quelque chose est, il ne peut pas suivre que quelque chose doive être, non plus qu'inversement de ce que quelque chose doit-être, il ne peut pas suivre que quelque chose est ${ }^{30}$.

L'on ne peut donc inférer d'un Sein un Sollen ${ }^{31}$, et inversement. Certes, Kelsen admet qu'il existe des relations entre être et devoirêtre, mais celles-ci ne peuvent transcender leurs deux modes respectifs d'existence spécifiques (Sein pour l'un; Sollen pour l'autre).

"possède une fonction essentiellement épistémologique " (Andràs Jakab, «Problèmes de la Stufenbaulehre. L'échec de l'idée d'inférence et les perspectives de la théorie pure du droit ", Droit et société, $\mathrm{n}^{\circ}$ 66, 2007/2, p. 420; Hans Kelsen, $T P D$, op. cit., p. 201). On retrouve la même analyse chez certains juristes italiens adeptes de la philosophie analytique du langage: «Le principe de vérification empirique opérant à un niveau métalinguistique comme critère de sélection des propositions admissibles ou non admissibles dans le système de la science, il n'est pas tenu de présenter les caractéristiques qu'il exige des propositions auxquelles il s'applique [...]. Les contradictions de la norme fondamentale peuvent s'expliquer et se résoudre de la même manière: l'origine de la contradiction se trouve dans le fait de référer la proposition qu'exprime la norme fondamentale à elle-même alors que cette proposition se situe à un niveau métalinguistique par rapport aux propositions du système de normes, ce qui signifie que les propositions qu'elle pose ne concernent pas son propre niveau, ne lui sont pas applicables à elle-même » (Uberto Scarpelli, op. cit., p. 48-49). Ainsi, la norme fondamentale n'a pas pour objet de fonder l'autorité d'un système de normes mais simplement d'en rendre possible l'étude (Voir Hans Kelsen, TPD, op. cit., p. 200). Dès 1928, Kelsen énonce d'ailleurs que «C'est précisément dans le difficile renoncement à une justification absolue, matérielle, dans le fait de se limiter avec abnégation au fondement purement hypothétique et formel de la norme fondamentale, que réside la marque distinctive essentielle du positivisme par rapport à la doctrine du droit naturel. Le positivisme et le relativisme épistémologique sont liés [...] » (Hans Kelsen, «La doctrine du droit naturel et le positivisme juridique », art. cité, p. 440).

30. Hans Kelsen, TPD, op. cit., p. 14.

31. Le Sollen, dans son sens juridique, intègre une signification plus large que ne le laisse entendre le verbe Sollen dans la langue allemande puisque, comme le souligne l'auteur de la Théorie pure du droit, Sollen renvoie également à "Dürfen» (avoir le droit de: permission), «Können» (avoir le pouvoir de : habilitation). «Car aussi bien que commander, une norme peut permettre et, en particulier, donner pouvoir » (ibid., p. 13). 
Kelsen, redevable à son élève et collègue Adolph Julius Merkl de l'introduction au sein de la théorie pure du droit de la « doctrine de la pyramide des normes " (Stufenbaulehre), explique grâce à elle le caractère dynamique de l'ordre juridique ${ }^{32}$ par l'idée d'inférence: chaque norme trouve le fondement de sa validité (c'est-à-dire sa positivité et même son existence, pour Kelsen) dans la norme qui lui est immédiatement supérieure. Ainsi, pour être valide, la loi « $\mathrm{L}$ » doit être produite conformément aux dispositions (essentiellement formelles) énoncées par la Constitution « $\mathrm{C} »$. L'acte de volonté (édiction de la loi « $\mathrm{L} »$ ) ne peut être considéré comme un acte de création de droit que si, et seulement si, la Constitution « $C$ » l'a prévu. Étant donné la structure normative de l'ordre juridique, il n'y a qu'une seule Constitution, de nombreuses lois, davantage encore de règlements, etc. Plus on descend dans la hiérarchie des normes, plus les normes sont nombreuses et concrètes. Chaque norme se trouve ainsi dans un rapport de validité (dit aussi « rapport selon l'ordre de production") avec les normes immédiatement supérieures et inférieures. Chaque norme conditionne la procédure de production (et la validité) de la norme inférieure, tout comme sa production et sa validité sont elles-mêmes conditionnées par la norme supérieure. Il apparaît donc, à la lumière de la Stufenbaulehre, que "l'origine de la validité d'une norme ne peut ainsi provenir que d'une autre norme ${ }^{33} »$.

Problème: l'idée d'inférence se trouve, dans la théorie pure du droit de Kelsen, mise en défaut par la loi de Hume. Et, cela, que cette idée implique d'inférer, soit d'un être un devoir-être (a) soit, au contraire, d'un devoir-être un être (b).

a. Si l'on ne peut inférer d'un être un devoir-être, ni d'un devoir-être un être, alors la création effective de la norme « $\mathrm{N}_{2}$ » selon les conditions de validité énoncées par la norme supérieure « $\mathrm{N}_{1}$ » constituent un être, duquel on ne peut inférer la validité de la norme « $\mathrm{N}_{2}$ », la validité étant un devoir-être.

32. Nous reprenons, pour le moment, l'assimilation entre les concepts d'« ordre juridique » et de "système juridique », qui peut être imputée à Kelsen, mais aussi à Herbert Hart, Norberto Bobbio, Jospeh Raz, Eugénio Bulygin, etc.

33. Andràs Jakab, art. cité, p. 411-447, en particulier p. 413. Comme le souligne Kelsen, «Le fondement de validité d'une norme est toujours une norme, ce n'est jamais un fait» (Hans Kelsen, TGDE, op. cit., p. 165). 
Reprenons l'exemple de la Constitution « $\mathrm{C} »$, qui énonce les conditions de validité de la loi « $\mathrm{L} »$. L'inférence de Kelsen est la suivante :

- contenu des dispositions de la Constitution « $\mathrm{C} »$ : une loi « $\mathrm{L} »$, pour être valide, doit être édictée conformément aux présentes dispositions de la Constitution " $\mathrm{C}$ »;

- la loi «L» est édictée conformément aux prescriptions de la Constitution «C» (fait ou Sein). Donc la loi «L» acquiert validité et, par conséquent, est valide (c'est-à-dire est un Sollen ou devoir-être). Ainsi, Kelsen tire d'un être (la production de la loi selon la procédure prescrite par la Constitution) un devoir-être ${ }^{34}$ (la validité de la loi ainsi

34. Cette relative confusion se retrouve à différents endroits des deux écrits majeurs de Kelsen, notamment la Théorie générale du droit et de l'État : « Tout acte de création du droit doit être un acte d'application du droit, il doit appliquer une norme qui lui est antérieure afin d'être un acte de l'ordre juridique ou de la communauté que cet ordre constitue» (p. 188); "Dans la mesure où la norme est établie grâce à l'acte [de volonté d'un organe], celui-ci est un acte créateur de droit [...]» (ibid.); «Si on appelle ces normes des normes juridiques, c'est parce qu'elles ont été créées par une autorité qui, en vertu de la constitution, a compétence pour créer le droit. [...]. Selon ce concept [d'inférence], est droit tout ce qui a été créé au terme de la procédure de création du droit prescrite par la constitution» (p. 176); « une norme qui exprime l'idée que quelque chose doit se produire - bien qu'il soit possible que cette chose ne se produise pas effectivement - est une norme "valide". [...] L'échec sera la conclusion inéluctable de toute entreprise visant à expliquer les normes juridiques au moyen de règles qui décrivent la conduite effective des hommes, sans recourir au concept de "devoir-être" » (p. 87). La mise en apposition de deux phrases, espacées de seulement deux pages, résumera à merveille la contradiction logique de Kelsen: «Le fondement de validité d'une norme est toujours une norme, ce n'est jamais un fait» (p. 165); "Une norme est une norme juridique valide parce qu'elle a été créée conformément à une règle précise» (p. 167). Or, la procédure de création - conforme ou non - relève du fait. Ainsi, d'un fait, Kelsen infère une norme. On pourrait arguer, pour réfuter cette première thèse, que celle-ci opère en réalité une confusion entre le discours prescriptif du droit et le discours descriptif de la science du droit. Le théoricien du droit, Kelsen, procéderait à une description d'un sollen, en formulant un sollen descriptif («la norme juridique est valide»). En parallèle, selon le discours prescriptif de l'ordre juridique «OJ», la norme juridique « N2 » n'est qualifiée comme «valide» que par l'organe « $\mathrm{O}$ » qui a à l'appliquer. Établissant la qualification juridique du fait (procédure de création de la norme « $\mathrm{N}_{2}$ » conforme à celle exigée par la norme « $\mathrm{N}_{1}$ »), le juge «J " pourrait énoncer que « $\mathrm{N} 2$ » est valide et appartient par conséquent à « $\mathrm{OJ}$ ». Néanmoins ce réagencement de l'argument kelsénien se heurte à la structure hiérarchique de l'ordre juridique avancée par Kelsen et Merkl. Car, dans cette hypothèse, la validité d'une norme " $\mathrm{N}_{2}$ » ne serait plus établie par la norme " $\mathrm{N}_{1}$ », hiérarchiquement supérieure, mais par la norme édictée par l'organe d'application, c'est-à-dire " $\mathrm{N}_{3}$ ", norme hiérarchiquement inférieure. Il y aurait alors un renversement de la «dynamique » du droit telle que la conçoit le juriste viennois (Voir Hans Kelsen, TGDE, op. cit., p. 178). En outre, un autre obstacle réside dans le domaine temporel de validité des 
produite). Or, la formulation de l'inférence qui respecterait la loi de Hume ne peut être que la suivante:

- contenu des dispositions de la Constitution «C»: "Si une loi "L" est édictée conformément aux présentes dispositions, elle doit être considérée comme valide ${ }^{35} »$;

- la loi «L» est édictée conformément aux prescriptions de la Constitution « $\mathrm{C}$ » (fait). Donc la procédure pour créer une loi « $\mathrm{L}$ » a effectivement été respectée (fait).

Mais il s'agit alors d'une particularité de l'être de la norme, distincte du devoir-être qu'implique la validité. En effet, pour Kelsen, « Puisque la validité d'une norme constitue un Sollen, et non un Sein, elle est quelque chose de différent de son efficacité, c'est-à-dire du fait de Sein que la norme est effectivement appliquée et obéie ou suivie, que le comportement humain qui y correspond se produit effectivement ${ }^{36}$ ». En outre, selon le juriste autrichien, qui fait une application stricte de la loi de Hume: «La conduite qui est et la conduite qui doit être ne sont pas identiques; certes, la conduite qui doit être ressemble à la conduite qui est ; mais une différence les sépare quand même : la circonstance - ou modalité - que l'une "est existante", alors que l'autre "doit exister". [...] Lorsque l'on dit: le Sollen tend à un Sein, la norme tend à provoquer une conduite effective, c'est à la conduite effective qui correspond au contenu de la norme que l'on pense [création de la loi selon la procédure prescrite dans le contenu de la Constitution, par exemple], au contenu du Sein, contenu qui ressemble à celui du Sollen [...] en raison du mode différent : Sein dans un cas, Sollen dans l'autre ${ }^{37}$." La loi de Hume parait donc fortement fragilisé d'un côté, Kelsen inférant d'un être un devoir-être.

En effet, si la création effective d'une loi (Sein) est conforme à la procédure prévue par la Constitution en son contenu (Sollen), ce fait ne peut toutefois être considéré comme un Sollen. Par conséquent, c'est

normes tel qu'établi par Kelsen: «Validité et effectivité des normes juridiques ne coïncident pas non plus dans le temps. Les normes juridiques entrent en vigueur avant même qu'elles ne deviennent effectives, c'est-à-dire avant même qu'elles ne soient suivies et appliquées» (Hans Kelsen, TPD, op. cit., p. 19).

35. Comme le souligne d'ailleurs Kelsen, il ne résulte pas du caractère prescriptif (au sens large) de la norme "que les organes de création du droit doivent nécessairement présenter les normes sous la forme d'énoncés hypothétiques de "devoir-être". Les éléments d'une norme peuvent [...] trouver leur expression dans des énoncés linguistiques fort variés. [Ainsi] Le législateur utilise fréquemment le temps du futur: le voleur "sera" puni de telle ou telle façon. [...]. L'expression "sera puni" n'est pas une prophétie [...] mais une obligation".

36. Ibid.

37. Ibid., p. 15. 
de manière tout à fait illogique que l'on présupposerait que l'opération de création a abouti à une norme valide (Sollen), puisque l'on ne peut inférer d'un être un devoir-être. L'acte qui résulte de cette opération de création n'est qu'un énoncé (et non une norme) dont la validité ne peut être affirmée.

b. Plus radical encore est l'échec souligné par Andràs Jakab qui permet d'entrevoir que, quand bien même Kelsen n'aurait pas inféré d'un être un devoir-être, il aurait tout de même opéré une autre erreur logique, en contradiction avec la loi de Hume, en inférant du devoir-être de la norme de création (norme prévoyant les conditions de validité d'une norme inférieure) l'être de la validité de la norme crée.

La création de la norme peut être résumée à travers les trois étapes suivantes :

1. «Si (succès de l'action de création de norme), alors (la loi [A] doit exister);

2. succès de la création de norme.

3. la loi $[\mathrm{A}]$ doit exister ${ }^{38}$.»

Or, Kelsen, à partir de l'idée d'inférence, tire de l'étape 2 une étape 3 bien différente :

3. La loi $[\mathrm{A}]$ existe (et non «la loi $[\mathrm{A}]$ doit exister»).

Ainsi, «de l'énoncé que $A$ doit être valide (ou doit exister), il en conclut que l'énoncé $A$ est valide (ou existe) ${ }^{39}$ ». Il suffit d'insérer dans le domaine conséquentiel de la règle de création ("alors...») la prescription d'un comportement physique pour voir plus clairement la rupture de la loi de Hume. L'exemple d'un feu de circulation peut servir ici d'exemple, certes schématique :

1. «Si (feu de circulation rouge), alors (l'automobiliste doit s'arrêter).»

2. Le feu est rouge.

3. L'automobiliste s'est arrêté (selon Kelsen). 
Kelsen nous invite donc à inférer d'un devoir-être (celui de la règle de création), l'être de la validité, c'est-à-dire son existence. Alors même qu'il n'est pas question de dérivation ou d'inférence, mais bien de probabilités. L'erreur logique, se double d'une contradiction: pour Kelsen et les partisans de la Stufenbaulehre, "la dérivation (l'inférence) de la validité exclut toute forme de validité basée sur la probabilité ${ }^{40} »$.

Dans les deux schémas ( $\mathrm{a}$ et $\mathrm{b}$ ) présentés ici, dont l'interprétation peut être cumulative (c'est-à-dire synchronique) ${ }^{41}$, l'échec de l'idée d'inférence apparaît nettement et emporte des conséquences relativement importantes : la Théorie pure du droit ne permet plus « de répondre aux questions de l'origine de la validité du droit et du fondement de l'unité de l'ordre juridique ${ }^{42}$ ». En effet, selon Kelsen, une norme juridique n'existe jamais indépendamment, mais uniquement parce qu'elle est reconnue comme valide par un système juridique donné. Le construit kelsénien de l'inférence, censé fonder l'unité de l'ordre juridique, parce qu'il réduit la validité à une simple question formelle et assimile de manière réductrice « existence » et "validité », ne peut rendre compte de la création du droit.

\subsubsection{Quelques conséquences envisageables}

C'est pourquoi il est tout à fait difficile de comprendre la conclusion d'A. Jakab qui, soulignant qu'il ne reste plus qu'à «prélever [les organes] de la Théorie pure du droit dont le cœur - la Stufenbaulehre - a définitivement cessé de battre ${ }^{43}$ ", mentionne parmi ces «organes" «la volonté d'expliquer les phénomènes juridiques à l'aide d'une structure conceptuelle unitaire ${ }^{44}$ ». Il semble plutôt que l'échec de l'idée d'inférence constitue également celui de la démonstration de l'unité du droit et/ou de la science du droit telle que (pré)conçue par Kelsen et les normativistes.

Expliquons-nous : c'est principalement de l'argument transcendantal néo-kantien (intégrant la distinction entre Sein et Sollen ainsi que la catégorie de l'imputation qui en découle) qu'est issue l'idée d'une

40. Ibid., p. 437.

41. Andràs Jakab s'en tient à un seul des deux aspects de l'échec de l'idée d'inférence présentés ici : celui selon lequel Kelsen infère d'un devoir-être un être (voir notamment Andràs Jakab, art. cité, p. 450). Il ne se prononce donc ni sur le bien-fondé du premier aspect, ni sur le caractère potentiellement cumulatif de ces deux entorses à la loi de Hume.

42. Ibid., p. 449 .

43. Ibid.

44. Ibid. 
inférence entre les normes, inférence basée sur un rapport de production. Autrement dit, la structure unitaire du système repose sur cette structure conceptuelle unitaire qu'est la validité conceptualisée sous la forme de l'inférence ${ }^{45}$. Or, l'idée d'inférence constitue un échec et les normes censées être acceptées comme valides ne peuvent l'être sur le seul fondement de cette structure conceptuelle unitaire. Dès lors, si l'on admet avec Sandrine Pina que «le coryphée de l'école viennoise établit [...] une auto-régulation du droit [, que] le droit est [selon cette école] autofondé par la norme fondamentale et [que] le système fonctionne sur lui-même ${ }^{46}$ ", alors l'échec de l'idée d'inférence implique nécessairement l'effondrement de la théorie de l'«autorégulation» du droit au sens où l'entend Kelsen ${ }^{47}$. L'unité du système juridique apparaît également rompue en ce qu'elle ne peut plus être démontrée à partir du concept de validité, entendu au sens restreint (kelsénien) de la validité formelle ${ }^{48}$. Pourtant, force est de reconnaître que les autorités juridiques

45. C'est du moins ainsi que Kelsen présente les choses.

46. Sandrine Pina, « La connaissance pure du droit et ses limites ", Communication présentée lors $\mathrm{du} \mathrm{VI}^{\mathrm{e}}$ congrès français de droit constitutionnel, congrès de Montpellier, AFDC, 9-11 juin 2005, atelier 4: "Quels outils théoriques pour comprendre le droit?", disponible sur http://www.droitconstitutionnel.org/congresmtp/atelier4.html (consulté le 9 septembre 2013), 23 p., en particulier p. 10.

47. Comme le souligne Kelsen, dans la Théorie pure du droit, "on a déjà évoqué à maintes reprises cette particularité que présente le droit de régler lui-même sa propre création. [...]. En accord avec le caractère dynamique de l'unité des ordres juridiques, une norme est valable [c'est-à-dire valide] si et parce qu'elle a été créée d'une certaine façon; celle que détermine une autre norme » (Hans Kelsen, TPD, op. cit., p. 224).

48. Comme le précise Kelsen, «L'ordre juridique n'est pas un système de normes placées toutes au même rang, mais un édifice à plusieurs étages superposés, une pyramide ou hiérarchie formée (pour ainsi dire) d'un certain nombre d'étages ou couches de normes juridiques. Son unité résulte de la connexion entre éléments qui découle du fait que la validité d'une norme qui est créée conformément à une autre norme repose sur celle-ci; qu'à son tour la création de cette dernière a été elle aussi réglée par d'autres, qui constituent à leur tour le fondement de sa validité; et cette démarche régressive débouche finalement sur la norme fondamentale, norme supposée. La norme fondamentale hypothétique - en ce sens - est par conséquent le fondement de validité suprême, qui fonde et scelle l'unité de ce système de création " (ibid.). Or, pour Kelsen, tout système juridique est avant tout un système dynamique, c'est-à-dire dont le principe d'unité repose sur la procédure de création du droit : «Les systèmes de normes qui se présentent comme des ordres juridiques ont pour l'essentiel un caractère dynamique. Une norme juridique n'est pas valable [valide] parce qu'elle a un certain contenu, [...] elle est valable parce qu'elle est créée d'une certaine façon, et plus précisément, en dernière analyse, d'une façon qui est déterminée par une norme fondamentale» (ibid., p. 197). 
de tout système juridique considèrent les normes formellement valides comme réellement valides et ce, dès leur édiction. Cette inadéquation fondamentale entre la théorie pure et la pratique, la première s'avérant impropre à expliquer la seconde, emporte une implication majeure et deux conséquences possibles à travers une alternative logique qu'il faut envisager ici en premier lieu.

\section{a. L'alternative, d'abord}

Option 1. Soit toute structure conceptuelle unitaire s'avère être un échec en ce qu'aucune ne parvient, pas plus que la structure conceptuelle kelsénienne, à rendre compte de l'objet «droit valide» sans contradiction manifeste ou simplification excessive. Dès lors, une structure conceptuelle plurielle s'avère inévitable et nécessaire pour répondre à l'objectif de la science du droit consistant à connaître et décrire son objet.

Option 2. Soit c'est uniquement la structure conceptuelle unitaire kelsénienne qui aboutit à un échec. Dans ce cas, ce n'est pas l'aspect unitaire de la structure conceptuelle qui doit être réexaminé, mais le fondement même de l'unité de l'objet droit, à travers une nouvelle structure conceptuelle. Il faut, en outre, se départir des présupposés kelséniens, y compris - et notamment - la distinction entre être et devoirêtre, au cœur de l'argument transcendantal néo-kantien, distinction permettant de postuler la catégorie de l'imputation comme une nécessité scientifique.

\section{b. Une implication majeure, ensuite}

Quelle que soit l'option retenue, au terme de la mise en exergue de cette alternative, il faut admettre l'effondrement du cœur de la théorie normativiste selon lequel le droit s'autorégule et constitue par conséquent une structure unitaire: ce n'est pas parce que le droit règle les conditions de sa propre création que le droit ainsi créé peut être considéré comme valide au sein du système juridique (c'est-à-dire existant - selon l'assimilation opérée par Kelsen entre existence et validité - en tant que devoir-être).

Cette assertion doit néanmoins être appréhendée de façon distincte en fonction du terme de l'alternative retenu:

- dans l'option 1, ce n'est pas seulement parce que le droit règle les conditions de sa création que le droit créé est considéré comme valide;

- selon l'option 2, ce n'est tout simplement pas parce que le droit règle ces conditions que le droit ainsi créé est considéré comme valide et peut donc être considéré comme «droit» (la propriété du droit 
étant d'être valide). La clôture normative du droit n'est donc pas mise en avant par la Théorie pure $d u$ droit mais, paradoxalement, en défaut.

Reste donc à savoir si les néo-normativistes ont apporté une solution convaincante à ce "grand mystère du droit ${ }^{49}$ " que constitue la validité juridique, au regard du terme de l'alternative retenu.

\subsection{Le néo-normativisme, constructivisme sans issue}

Plusieurs auteurs ont tenté de réduire les apories kelséniennes tout en s'inscrivant dans la théorie pure du droit. Comme le souligne Andràs Jakab, ces "partisans contemporains de la théorie pure du droit affirment qu'ils continuent à la développer, alors qu'en réalité ils la reconstruisent $\mathrm{t}^{50} »$. On a donc déjà un indice sur l'aspect constructiviste d'une telle entreprise. Toutefois le constructivisme néo-normativiste est parfois pleinement assumé, au contraire du constructivisme normativiste. Ainsi, Otto Pfersmann, représentant le plus célèbre en France du courant néo-normativiste (et qui accepte l'ensemble des postulats de Kelsen ${ }^{51}$ ), critique l'expression de "concurrence entre les systèmes juridiques " pour le motif principal suivant: «Premièrement, les systèmes ou ordres juridiques ne sont pas des données naturelles, mais des constructions intellectuelles par lesquelles des ensembles d'énoncés prescriptifs sont

49. Andràs Jakab, art. cité, p. 449.

50. Ibid., p. 442.

51. Tout en y apportant quelques améliorations substantielles, relatives à la pluralité des hiérarchies normatives (Voir notamment Otto Pfersmann, "Hiérarchie des normes », dans Denis Alland et Stéphane Rials, op. cit., p. 779-783; «La constitution comme norme" (sauf le dernier chapitre), dans Louis Favoreu et al., Droit constitutionnel, (13 éd.), Paris, Dalloz, «Précis», 2010, p. 53-117), aux rapports de systèmes (Otto Pfersmann, «La production des normes: production normative et hiérarchie des normes », dans Michel Troper et Dominique Chagollaud (dir.), Traité international de droit constitutionnel, Paris, Dalloz-Sirey, «Traités », août 2012, t. II, «Distribution des pouvoirs», p. 483-528, en particulier p. 514-526; « La primauté: double, partiellement directe, organiquement indéterminée, provisoirement fermée», CCC, $\mathrm{n}^{\circ} 18$, juillet 2005, 5 p.), mais sans modification substantielle du substrat théorique kelsénien. Voir encore Otto Pfersmann, "Qu'entend-on exactement par l'expression "concurrence des systèmes juridiques" ", Revue de la recherche juridique. Droit Prospectif, 125 2008/22 HS, p. 2595-2601. 
constitués comme objectivement normatifs pour les besoins de l'analyse $^{52}$.» On est donc bien loin de «l'engagement positiviste, qui consiste à décrire une réalité distincte du sujet de la connaissance ${ }^{53}$ ». On notera d'ailleurs qu'O. Pfersmann affirme sa démarche comme "constructiviste $^{54} »$ par nature ${ }^{55}$.

Reste que les grands auteurs néo-normativistes contemporains n'ont pas résolu le problème de l'échec de l'idée d'inférence, malgré la mise en avant d'une pluralité de hiérarchies normatives (1.2.1). Ils ont même parfois accentué la réduction opérée par Kelsen dans la définition même du droit positif, en ôtant une ou plusieurs des fondations faisant tenir l'édifice kelsénien, notamment la norme fondamentale (1.2.2). Le constat est donc négatif: les solutions (constructives) normativistes ne parviennent pas à résoudre les apories du constructivisme kelsénien, donnant à voir ce dernier comme sans issue.

52. Otto Pfersmann, art. cité, p. 2596. Le problème, toutefois, de cette explicitation du caractère constructiviste de l'analyse juridique est qu'elle est censée s'appliquer à l'ensemble des définitions desdits «systèmes juridiques». Or, Otto Pfersmann réduit ce concept à son expression normativiste, excluant l'ensemble des conceptions postnormativistes (Santi Romano, mais aussi Paul Amselek, Jean Combacau, Joseph Raz, Ronald Dworkin) [voir, pour une synthèse des doctrines post-normativistes des systèmes juridiques, Charles Leben, "De quelques doctrines de l'ordre juridique ", Droits, ${ }^{\circ}$ 33, 2001, p. 25-29].

53. Michel Troper, Pour une théorie juridique de l'État, op. cit., p. 161.

54. Ainsi Otto Pfersmann affirme: «Les principaux reproches que l'on a coutume d'adresser à une démarche comme la nôtre sont les suivants: 1 . il s'agirait d'un scientisme qui ne tient pas compte de la "réalité" et qui ne pourrait pas le faire parce qu'il la plierait d'abord à son image. À cela il faut répondre que toute approche scientifique d'une réalité est tributaire de prémisses théoriques. [...] L'avantage des théories constructivistes est de rendre leurs prémisses explicites et par là ouvertement critiquables. À l'inverse une théorie qui prétend être ancrée dans la "réalité" postule [...] une forme de réalisme métaphysique [...]. Ce qui nous semblerait curieux, ce serait qu'on nie la possibilité ou la légitimité de construire un objet de telle manière qu'il puisse être étudié de manière scientifique, même s'il existait une pratique séculaire qui aurait également un objet désigné par le même terme pour champ d'investigation et de manipulation sans viser une connaissance spécifiquement scientifique» (Otto Pfersmann, «Temporalité et conditionnalité des systèmes juridiques ", Revue de la recherche juridique. Droit prospectif, ${ }^{\circ}{ }_{56}$ 6, 1994-1, p. 224, note 4).

55. Ce qui pourrait sembler paradoxal puisque il admet ainsi un emprunt à la sociologie tout en condamnant tout syncrétisme des méthodes, tout cela dans la même note de bas de page (ibid.) 


\subsubsection{La pluralité des hiérarchies normatives: complication sans solution}

L'un des auteurs les plus reconnus de l'école viennoise, Robert Walter, a tenté une restructuration de la hiérarchie normative.

Ainsi, dès $1964^{56}$, il réintroduisait l'idée dégagée par A. Merkl ${ }^{57}$ d'une hiérarchie selon la "force dérogatoire ${ }^{58}$ ». À l'inverse de la hiérarchie selon l'ordre de production (rapport de validité entre les normes), la hiérarchie selon l'ordre dérogatoire fait état d'un rapport de conformité : « une norme $\mathrm{A}$ est supérieure à une norme $\mathrm{B}$ si la norme $\mathrm{A}$ peut déroger à la norme $\mathrm{B}$ et si l'inverse est exclu ${ }^{59}$. ${ }^{2} \mathrm{La}$ "dérogation » étant ici entendue comme "l'aptitude d'une norme juridique à abroger ou à limiter la validité d'une autre norme juridique ${ }^{60} \%$. Ainsi, alors que le contrôle de constitutionnalité a priori relève d'une technique préventive visant "à rendre plus difficile la production de normes entachées de défauts ${ }^{61}$ ", ne fondant donc pas de nouvelle hiérarchie, le contrôle par voie de QPC, a posteriori, participe, lui, de la complétion d'une hiérarchie selon la force dérogatoire. On peut, à l'instar d'Otto Pfersmann et de l'ensemble des néo-normativistes, souligner que cette hiérarchie selon la force dérogatoire fait partie des remèdes normatifs relatifs au phénomène inévitable de la " concrétisation ». Cette concrétisation est liée au caractère dynamique des ordres juridiques et consiste en ce que, plus on descend dans la hiérarchie des normes selon l'ordre de production, plus les normes sont susceptibles de comporter des défauts, étant donné le caractère essentiellement formel des conditions de validité. Aux côtés de la hiérarchie selon la force dérogatoire existe également une hiérarchie selon la primauté d'application, liée à l'introduction dans l'ordre juridique de normes privant la concrétisation de son effet en interdisant l'application de normes valides au profit d'autres normes ${ }^{62}$.

56. Robert Walter, Der Aufbau des Rechtsordnung, Vienne, Manz, 1964, p. 55 et suiv.

57. Adolph Merkl, "Prolegomena einer Theorie des rechtligen Stufenbaues ", dans Alfred Verdross (dir.), Gesellschaft, Staat und Recht. Untersuchungen zur reinen Rechtslehre. Festschrift Hans Kelsen zum 5o. Geburtstag gewidmet, Vienne, Springer, 1931, p. 1350.

58. Otto Pfersmann, «Hiérarchie des normes», art. cité, p. 781.

59. Andràs Jakab, art. cité, p. 411-447, en particulier p. 438.

60. Theo Öhlinger, Der Stufenbau der Rechtsordnung. Rechtstheoretische und ideologische Aspekte, Vienne, Manz, 1975, p. 22, traduit dans Andràs Jakab, art. cité, p. 439.

61. Otto Pfersmann, art. cité, p. 781.

62. Le contrôle de conventionalité pratiqué en France correspond à la mise en avant d'une telle hiérarchie puisqu'il est fondé sur l'article 55 de la Constitution de 1958 qui dispose que les traités ratifiés ou approuvés et publiés ont «une autorité 
Toutefois, force est d'admettre que ces deux hiérarchies normatives ne «peuvent [...] prétendre à remplacer la Stufenbaulehre ${ }^{63}$ » puisque, comme le souligne O. Pfersmann lui-même, «la hiérarchie selon l'ordre de production fonde les autres [...]. [En outre], quelles que soient la complexité et la subtilité des calculs de défauts, il est impossible d'éliminer la non-conformité des règles valides puisqu'il va toujours falloir produire une nouvelle norme valide afin de déterminer si une norme attaquée est, ou non, entachée de défauts ${ }^{64}$ ».

Dit autrement, le problème de la création de normes valides, qui ne se trouvait pas résolu par Kelsen, ne l'est pas non plus par les néonormativistes, qui se heurtent également à l'échec de l'idée d'inférence.

\subsubsection{Le retrait de la norme fondamentale: simplification sans cohérence}

Une autre tentative a été tentée pour rendre sa cohérence à la Théorie pure du droit: l'abandon de la "norme fondamentale" allié à la conservation des autres thèses kelséniennes. Un tel retrait ne paraît toutefois pas en mesure de répondre à l'objectif assigné, et tend au contraire à accentuer l'aspect réducteur du constructivisme kelsénien.

Ainsi, l'exposé des thèses kelséniennes est repris quasiment tel quel: «On considère [...] que le droit est un ordre contraignant de la conduite humaine, et qu'il correspond à un ensemble de normes organisées selon une certaine hiérarchie, cet ensemble étant clos et indépendant des autres ordres sociaux. En raison de ce cloisonnement, le droit fonctionne, en quelque sorte, en "autarcie" ou de façon autorégulé: est juridique ce que le droit qualifie de tel ${ }^{65} \ldots »$

supérieure à celle des lois sous réserve, pour chaque accord ou traité, de son application par l'autre partie» (art. 55 C.) La loi est ainsi privée de son application, sans être formellement annulée. Une telle hiérarchie, pour autant qu'elle soit mise en œuvre régulièrement, comme c'était le cas avant l'introduction de la QPC en France, explique par exemple la neutralisation du droit national.

63. Andràs Jakab, art. cité, p. 442.

64. Otto Pfersmann, art. cité, p. 781-782.

65. Jean-Philippe Derosier, Les limites constitutionnelles à l'intégration européenne. Étude comparée: Allemagne, France, Italie, thèse pour l'obtention du grade de docteur en droit, sous la direction du professeur Otto Pfersmann, 14 décembre 2010, p. 9 (pagination non définitive). 
De même, l'explicitation du concept de validité est opérée par renvoi direct aux écrits de Kelsen ${ }^{66}$ et en soulignant que «l'expression "une norme valide" constitue un pléonasme car, s'il y a norme, cette dernière est valide et, si elle n'est pas valide, il n'y a pas de norme ${ }^{67}$ ». La validité d'une norme est, également, supposée découler de ce que qu'elle "a été produite selon les règles de production prévues par cet ordre juridique ${ }^{68} »$. Et « la hiérarchie des normes est inhérente à tout ordre juridique: il s'agit d'une théorie qui en explique le fonctionnement [celui de l'ordre juridique] et non d'une propriété contingente qui serait intégrée dans l'ordre juridique par certaines normes de cet ordre juridique $»$.

Trois remarques peuvent être faites :

- D’une part, les conceptions néo-normativistes n'apparaissent pas unifiées quant à l'aspect constructiviste de la Théorie pure $d u$ droit. Alors que O. Pfersmann admet que les systèmes juridiques sont des " constructions intellectuelles par lesquelles des ensembles d'énoncés prescriptifs sont constitués comme objectivement normatifs pour les besoins de l'analyse ${ }^{69}$ ", d'autres affirment que ce n'est pas l'analyse qui fonde la clôture normative du système mais que le droit correspond à un "ensemble étant clos et indépendant des autres ordres sociaux : en raison de ce cloisonnement, le droit fonctionne en quelque sorte en "autarcie" ".

- En deuxième lieu, et cela rejoint le point précédent, la hiérarchie des normes est définie à la fois comme «inhérente à tout ordre juridique » et comme "théorie qui explique le fonctionnement du système juridique». Or, on ne voit guère comment une théorie peut être inhérente à tout ordre juridique, autrement dit comment une théorie peut être une réalité effective. Elle est, ou une construction intellectuelle, ou une réalité naturelle. Mais affirmer que la propriété d'une théorie est d'être inhérente à la réalité constitue une aporie irréductible.

- Enfin, et c'est le point qui nous intéressera le plus, on retrouve le concept d'inférence:

66. «Nous entendons par "validité" l'existence spécifique des normes. Lorsque nous disons qu'une norme est valide, nous admettons qu'elle existe ou, ce qui revient au même, qu'elle a "force d'obligation" pour ceux dont elle régit la conduite " (Hans Kelsen, TGDE, op. cit., p. 81, cité par Jean-Philippe Derosier, op. cit., note 32, p. 14).

67. Jean-Philippe Derosier, op. cit., p. 14.

68. Ibid.

69. Otto Pfersmann, «Qu'entend-on exactement par l'expression “concurrence des systèmes juridiques" ", art. cité, p. 2596. 
Apparaît alors un lien entre les normes d'un même ordre juridique : il s'agit d'un rapport de validité ou de production puisque certaines normes sont valides dès lors qu'elles sont produites selon les conditions fixées par d'autres normes ${ }^{70}$.

Dit autrement, c'est bien d'un être (production effective des normes selon les conditions fixées par d'autres normes) qu'est inféré un devoirêtre (une norme valide au sens pléonastique relevé par l'auteur). Pourtant, tout comme chez Kelsen, la distinction Sein/Sollen est clairement affirmée: "En ce sens, une norme est un "devoir-être" (Sollen) et non un "être" (Sein) : elle exprime ce qui doit être, un comportement qui doit (ou peut) être suivi, mais elle ne signifie pas que ce comportement est (ou sera) effectivement suivi. Par conséquent, elle se distingue de l'acte de volonté lui-même [la production conforme aux conditions de validité prévues par la norme supérieure], elle ne fait que le traduire sur le plan juridique car cet acte juridique n'opère pas sur le plan du droit (du devoir-être) mais du fait (de l'être $)^{71}$."

Tout comme le normativisme, force est de constater que le néonormativisme n'a donc pas pour objet des normes juridiques au sens où ces deux composantes du même courant les conçoivent. $\mathrm{Ou}$, du moins, seule la Constitution pouvait apparaître comme une norme valide puisque fondée, non pas sur un critère de production, mais sur une supposition. C'est pourquoi le retrait, par certains néo-normativistes, de la norme fondamentale, et l'affirmation subséquente ${ }^{72}$ «que la (première) Constitution n'est elle-même ni valide, ni non valide ${ }^{73}$ " fait

70. Jean-Philippe Derosier, op. cit., p. 14.

71. Ibid., p. 12.

72. Affirmation reprise du courant réaliste (Riccardo Guastini) à travers un syncrétisme certain et tout à fait contraire à l'affirmation de la pureté de la théorie du droit professée par Kelsen et réaffirmée dans les études néo-normativistes.

73. Riccardo Guastini, «La structure du système juridique », dans Leçons de théorie du droit constitutionnel, traduit et présenté avec ajouts par Véronique ChampeilDesplats, Paris, Dalloz, "Rivages du droit», 2010, p. 51, cité par Jean-Philippe Derosier, op. cit., note en bas de page, p. 12: «On rejoint ainsi l'hypothèse du professeur Riccardo Guastini qui, discutant l'hypothèse de la norme fondamentale, admet la parfaite logique de la théorie de Kelsen [souligné par nous] mais demande "pourquoi chercher un fondement à la validité de la Constitution dans une norme supplémentaire ni valide, ni non valide? De ce point de vue, la stipulation de la norme fondamentale s'avère totalement superflue: elle est une duplication inutile de la Constitution". " C'est donc l'économie de moyens, ou la recherche de la simplicité, qui fonde ce retrait chez Riccardo Guastini, alors pourtant qu'il reconnaît la "parfaite logique de la théorie de Kelsen». Où l'on voit que le "superflu » permettait tout de même au constitutionnaliste d'étudier une norme et non une supposition ou une fiction. En outre, il paraît évident que la suppression de la 
de l'objet « droit » analysé en ces termes néo-normativistes spécifiques un objet purement et entièrement fictif - et non, comme on serait pourtant tenté de la croire, normatif. En outre, cette opération de retrait ne peut être réalisée qu'en contradiction avec les principes méthodologiques fondamentaux de la Théorie pure $d u$ droit, notamment le principe d'imputation, et l'affirmation que l'on ne peut inférer d'un être un devoirêtre (c'est-à-dire du fait, la Constitution). En effet, se trouve énoncée l'idée que «sa validité [celle de la Constitution historiquement première] ne peut être recherchée que dans un acte révolutionnaire ${ }^{74} »$ : le devoirêtre (Sollen) de la Constitution doit être recherchée dans un fait (Sein).

Toutefois, cette reconstruction de la théorie pure ne parvient pas à se détacher de l'attractivité de l'œuvre de Kelsen puisqu'il est parallèlement affirmé que «la validité de la norme juridique suprême d'un ordre juridique [c'est-à-dire la Constitution] doit simplement être admise ${ }^{75}$ ». Dit autrement, la validité de la Constitution repose sur un présupposé. Dès lors, que l'on qualifie ce présupposé de «norme fondamentale » ou de "règle d'admission », il n'en reste pas moins extrajuridique et, par conséquent, totalement similaire à la «norme fondamentale » qu'il est pourtant supposé évincer. En effet, la norme fondamentale de Kelsen ne pose pas autre chose que «la validité de la Constitution doit être admise $^{76}$ ». La formulation de ce présupposé, selon lequel « la validité de la norme suprême d'un ordre juridique doit simplement être admise", est d'ailleurs sans équivoque puisque elle recèle un devoir-être, un Sollen fondateur.

L'on voit donc que les théories normativistes et néo-normativistes relèvent de constructions élaborées mais, reposant sur des fondations réductrices, elles n'échappent pas pour autant à une forte contradiction,

catégorie de la «norme fondamentale» ne pose pas de problème au réaliste analysant le droit comme un ensemble de faits puisque « cette conception du droit rompt avec l'ontologie normativiste de Kelsen selon laquelle les normes juridiques seraient des entités idéelles relevant d'un Sollen qui échappe au monde des faits " (Véronique Champeil-Desplats, "Présentation », dans Riccardo Guastini, Leçons de théorie du droit constitutionnel, op. cit., p. 14).

74. Jean-Philippe Derosier, op. cit., p. 19.

75. Ibid., p. 19.

76. «Si [...] l'on renonce à rapporter la validité de la Constitution et la validité des normes créées en conformité de cette Constitution à une norme qui aurait été posée par une autorité méta-juridique telle que Dieu ou la Nature, il n'y a qu'une seule réponse possible: la validité de cette Constitution, son caractère de norme obligatoire doivent être supposés, admis comme hypothèses...» (Hans Kelsen, TPD, op. cit., p. 200). 
assurant être les seules méthodes normatives ou " juridiques " mais sans jamais parvenir à démontrer l'existence du droit (puisqu'assimilée par Kelsen à sa validité). Cette problématique est liée à un aspect du constructivisme précédemment évoqué : il repose sur un projet réductionniste.

\section{Un projet commun réductionniste, fondement de la fragilité du construit (néo)normativiste}

Ce projet commun au normativisme et au néo-normativisme est celui de la description d'un droit unitaire et cohérent. Certains ont affirmé qu'au sein de la Théorie pure du droit, la pureté est première, l'unité est seconde ${ }^{77}$. Toutefois, cette assertion n'est guère satisfaisante, car encore faut-il comprendre le double rapport - logique et causal - qui unit ces deux exigences: c'est parce que le droit est un objet représenté comme unique (une unité) qu'il est pur; et c'est parce que le droit est un objet unique et pur qu'il ne peut être décrit que par une méthode unique et pure. Pour reprendre le vocabulaire kelsénien, l'on peut dire que l'unité du droit est la condition tant per quam que sine qua non de sa pureté. Elle est le moyen pour atteindre cette finalité que constitue la «pureté ». En cela, l'unité de l'objet «droit » est la source de la méthode kelsénienne, considérée pour cette raison par les (néo)normativistes comme «seule méthode juridique ». Reste alors à démontrer cette unité. On peut distinguer entre la métathéorie kelsénienne, qui prescrit à la science du droit de décrire de façon à conférer au droit une unité dont il n'est pas forcément doté initialement (2.1), et la théorie kelsénienne, description de la structure d'un droit reconstruit comme unitaire (2.2).

\subsection{L'objet de la science du droit : conférer au droit une unité}

Le dogme de l'unité de l'objet scientifique trouve son fondement dans l'approche positiviste de la fin du XIX ${ }^{\mathrm{e}}$ siècle : le phénomène doit trouver à s'expliquer par lui-même (2.1.1). Kelsen intègre pleinement ce présupposé méthodologique et attribue à la science juridique le rôle de gardienne de l'unité logique de l'objet droit (2.1.2), l'implication

77. «Ces exigences de scientificité [kelséniennes] imposées à la science juridique se nourrissent à quatre sources notamment. [...]. La première de ces sources consiste dans la pureté [...]. Le deuxième mot clé est celui d'unité - à savoir l'unité exigée par Kelsen de la connaissance juridique » (Matthias Jestaedt, «La science comme vision du monde: science du droit et conception de la démocratie chez Hans Kelsen ", dans Olivier Jouanjan (dir.), Hans Kelsen. Forme du droit et politique de l'autonomie, Paris, PUF, « Débats philosophiques », août 2010, p. 181-184). 
majeure de cette métathéorie kelsénienne, contrairement à ce qui est souvent affirmé, étant de faire de la science du droit une science prescriptive (2.1.3).

\subsubsection{L'unité de l'objet scientifique ou l'explication du phénomène par... le phénomène}

Outre la reprise de la distinction humienne entre Sein et Sollen, dont on a vu l'importance au sein des thèses kelséniennes, Hans Kelsen s'inspire du mouvement positiviste en tant que « doctrine de la connaissance qui postule qu'il est préférable de renoncer aux considérations spéculatives sur l'essence ou la finalité d'un objet afin de le restituer dans sa factualité de donné d'expérience ${ }^{78}$ ». Auguste Comte avait clairement affirmé les progrès de l'évolution scientifique, décrivant cette dernière comme franchissant systématiquement trois périodes successives: la période théologique, où les raisons des phénomènes sont rapportées à des volontés occultes et anthropomorphiques; la période métaphysique, spéculative et tournée vers la raison d'être des phénomènes, les expliquant désormais par des principes abstraits (on pense ici au «vitalisme social » d'Hauriou ${ }^{79}$ ); la période positive, marquée par une science

78. Éric Maulin, «Positivisme», art. cité, p. 1171.

79. Maurice Hauriou, « La théorie de l'institution et de la fondation (essai de vitalisme social) ", dans Aux sources du droit: le pouvoir, l'ordre et la liberté, Paris, Bloud et Gay, "Cahiers de la nouvelle Journée ", $\mathrm{n}^{0} 23,1933$, p. 89-128. Selon Maurice Hauriou, «une institution est une idée d'œuvre ou d'entreprise qui se réalise et dure juridiquement dans un milieu social» (art. cité, p. 96); Il faut alors distinguer les «institutions-personnes", où «le pouvoir organisé et les manifestations de communion des membres du groupe s'intériorisent dans l'idée de l'œuvre» (ibid.), des « institutions-choses», dont le droit fait partie, qui ne sont pas, contrairement aux premières, intériorisées «dans le cadre de l'idée de l'œuvre» (art. cité, p. 97). "Toute corps constitué [l'] est pour la réalisation d'une œuvre ou d'une entreprise " (art. cité, p. 98). L'idée de l'œuvre à réaliser mérite donc le nom d'«idée directrice de l'entreprise» (ibid.). Mais ce concept d'«idée directrice» apparaît, chez Hauriou, relativement obscur: d'une part, l'idée directrice comprend « une part d'indéterminé et de virtuel qui porte au-delà de la fonction » (art. cité, p. 99); d'autre part, bien qu'Hauriou considère qu'une «idée directrice» a inévitablement une « existence positive», il ne renseigne pas le lecteur sur l'origine possible d'une telle idée, sinon par des considérations fort abstraites: «On doit se demander si cette nature objective de l'idée [directrice] est originaire et foncière. [...]. Du moment que les idées passent d'un individu à l'autre, elles doivent avoir dès le début, une nature objective. En réalité, il n'y a pas des créateurs d'idées, il y a seulement des trouveurs » (op. cit., p. 101). «Sans doute c'est interpréter en un sens vitaliste la célèbre «idée directrice» de Claude Bernard [au sens de force vitale créatrice], et cela retentit jusqu'à la biologie, mais, justement, le vitalisme a encore en biologie des partisans 
tournée vers les conditions d'existence de phénomènes, rompant avec toute idée de cause première ou finale, source ou produit inexplicable des phénomènes observés: ainsi, «le phénomène ne peut être expliqué que par le phénomène ${ }^{80}$ ».

On retrouve chez Kelsen la volonté d'inscrire le droit dans cette troisième et ultime phase: "Deux décades se sont déroulées depuis que j'ai commencé d'entreprendre une "théorie pure du droit"; [...] c'est-à-dire, une théorie du droit épurée de toute idéologie politique et de tous les éléments ressortissant aux sciences de la nature, consciente de son individualité, qui est liée à la légalité propre de son objet. Dès les débuts de cette entreprise, j'ai eu ce but présent à l'esprit : élever la science du droit [...], qui [...] se perdait presque complètement dans le raisonnement de politique juridique, au niveau et rang d'une véritable science $^{81} \ldots$ »

Et, en effet, dès sa thèse soutenue en 1911, Kelsen rejette tout "syncrétisme des méthodes ${ }^{82}$ ", affirmant vouloir «saisir la pureté de la théorie - entendue comme indépendance du droit - comme objet de la connaissance scientifique d'après deux directions différentes: d'abord contre les prétentions des considérations dites sociologiques voulant s'emparer du droit comme une part de la réalité naturelle d'après une méthode scientifique causale [où l'on comprend mieux la nécessité du principe d'imputation], et également contre les théories du droit naturel qui [ramènent la théorie du droit] à des postulats éthico-politiques ${ }^{83}$ ".

et puis le fait est là : projetée dans le plan social, dans la réalité du phénomène corporatif, l'idée directrice apparaît objective, c'est elle qui agit sur les adhérents, c'est sa mystique qui entraîne les foules » (op. cit., p. 109). Bien que la théorie vitaliste de Maurice Hauriou représente un apport majeur en ce qu'elle permet la mise en exergue de la part de créativité et d'inventivité dans l'action des autorités de création et d'application du droit, elle repose avant tout sur une approche idéaliste (voir, pour une lecture similaire, Neil MacCormick et Ota Weinberger, Pour une théorie institutionnelle du droit. Nouvelles approches du positivisme juridique, trad. Odile Nerhot et Philippe Coppens, Paris, LGDJ, «La pensée juridique moderne », 1992, p. 29-31). Nous reviendrons sur l'institutionnalisme de Maurice Hauriou lors de la définition positive de notre néo-institutionnalisme.

80. Éric Maulin, «Positivisme», art. cité, p. 1172.

81. Hans Kelsen, «Préface de la première édition», dans id., TPD, op. cit., p. 3.

82. Hans Kelsen, Hauptprobleme der Staatsrechtslehre, op. cit., p. 53, 294, 296, 468. Voir notamment Matthias Jestaedt, art. cité, p. 185. On retrouve, au titre premier de la Théorie pure du droit ce souci d'éviter « un syncrétisme de méthodes qui obscurcit l'essence propre de la science du droit et qui rend floues et vagues les bornes qui lui sont assignées par la nature de son objet : le droit» (Hans Kelsen, TPD, op. cit., p. 9).

83. Hans Kelsen, "Préface" à la $2^{\text {de }}$ éd. des Problèmes fondamentaux de la théorie juridique de l'État développés à partir de la théorie de la proposition juridique, cité 
Le projet de Kelsen est «avant tout un projet méthodologique. [...] Il entend construire une science où le juriste se borne à connaître son objet $^{84}$ ». Mais Kelsen lui-même admet à d'autres moments "que la science du droit, comme toute science, doit constituer son objet ${ }^{85}$ » : en effet, il souligne que, « conformément à la théorie de la connaissance de Kant, la science du droit a, de même que toute connaissance, un caractère constitutif, et qu'elle "créé" donc son objet en tant qu'elle le comprend comme un tout présentant une signification, un tout intelligible ${ }^{86}$ ». Malgré l'atténuation portée à cette affirmation par Kelsen, énonçant que "cette "création" n'a qu'un caractère purement intellectuel [, que] c'est seulement au regard de la théorie de la connaissance que l'on peut parler ici de création ${ }^{87}$ ", l'on ne peut que constater l'influence des conceptions méta-scientifiques ${ }^{88}$ kelséniennes sur la représentation de son objet d'étude. Alors que Matthias Jestaedt assure que l'unité affirmée par Kelsen est "l'unité de la connaissance juridique ${ }^{89}$ " et non celle de son objet, Kelsen lui-même affirme que cette connaissance doit demeurer "à l'intérieur des frontières que lui tracent l'objet et la méthode ${ }^{90}$ ». Cette circularité nous ramène inexorablement vers le présupposé positiviste selon lequel «le phénomène ne peut être expliqué que par le phénomène ${ }^{91}$ ». Affirmer l'unité et donc l'autonomie du droit,

et traduit par Sandrine Pina, «Hauptprobleme der Staatsrechtslehre, les fondements d'une théorie pure du droit », Jurisprudence. Revue critique, t. I, 2010, p. 61-69, en particulier p. 64.

84. Sandrine Pina, art. cité, p. 64 (souligné par nous).

85. Michel Troper, Pour une théorie juridique de l'État, op. cit., p. 167.

86. Hans Kelsen, TPD, op. cit., p. 80.

87. Ibid.

88. On peut, avec Vittorio Villa, définir la métascience comme une activité tentant de fournir des modèles explicatifs « des aspects principaux de la connaissance scientifique, c'est-à-dire d'effectuer une reconstruction critique de la science» (Vittorio Villa, La Science du droit, trad. Odile et Patrick Nerhot, Paris, LGDJ, «La pensée juridique moderne», 1991, p. 22). Pour Thomas Kuhn et Wolgang Stegmüller, cette reconstruction critique se situerait à mi-chemin entre la description entre la description et la prescription, en ce que toute méta-analyse critique des analyses scientifiques comporte nécessairement une part de prescription, liée à la manière dont le scientifique devrait procéder (voir Thomas Kuhn, "Reflections on why critics », dans Criticism and Growth of Knowledge, Imre Lakatos et Alan Murgrave (dir.), Presses de Cambridge, 1979, p. 237; Wolfgang Stegmüller, The Structure and Dynamics of Theories, New York, Heidelberg, 1976, p. 259).

89. Matthias Jestaedt, art. cité, p. 184.

90. Hans Kelsen, Das Problem der Souveränität und die Theorie des Völkerrechts. Beitrag $z$ u einer reinen Rechtslehre, ( $1^{\text {re }}$ éd. : 1920), traduit et cité par Matthias Jestaedt, art. cité, p. 182.

91. Éric Maulin, «Positivisme», art. cité, p. 1172. 
c'est affirmer l'unité et l'autonomie de la science du droit. La "pureté » constitue ici une «régurgitation» du monisme méthodologique lié au néo-positivisme autant que la traduction en droit de l'influence néokantienne ${ }^{92}$. C'est bien «pour assurer l'autonomie et la pureté du droit [de l'objet donc, et non seulement de la science qui l'étudie], [que] Kelsen a introduit le concept de norme fondamentale, fondation néokantienne permettant de clôturer l'ordre juridique sur lui-même ${ }^{93}$ ". Voire de le créer, puisque, comme le souligne Kelsen, "L'idéal de justice n'a en fin de compte d'autre sens que la norme fondamentale hypothétique du positivisme critique, dont la fonction est de constituer le matériel juridique empirique en un ordre ${ }^{94}$ », ou encore de "façonner [ce] matériel juridique empirique en un ordre intelligible, c'est-à-dire dépourvu de contradictions ${ }^{95} "$.

Pour reprendre la formule de Kelsen, "l'unité de la position de connaissance exige une approche moniste ${ }^{96} »$. Formule qui indique, comme le souligne A. Jakab, que «si l'on fait le choix d'adopter une méthode juridique unique, l'objet de connaissance doit lui aussi être

92. Kelsen lui-même affirmant reprendre la théorie de la connaissance de Kant et l'on a vu précédemment l'influence décisive de l'argument transcendantal néokantien dans la Théorie pure du droit (voir notamment Hans Kelsen, Théorie pure du droit, op. cit., p. 67 et suiv., 80, 102, 106 et suiv., 113, 125, 201, 205). Ce constat d'une influence néo-kantienne est du reste largement accepté (voir Matthias Jestaedt, art. cité, p. 18; Robert Alexy, Lukas H. Meyer, Stanley Paulson et Gerhard Sprenger (dir.), Neukantianismus und Rechtsphilosophie, Baden-Baden, Nomos, 2002, p. 223-251; Simone Goyard-Fabre, «L'inspiration kantienne de Hans Kelsen", Revue de métaphysique et de morale, vol. 83, n 2, 1978, p. 204-233 et «De l'idée de norme à la science des normes: Kant et Kelsen », dans Paul Amselek (dir.), Théorie du droit et science, Paris, PUF, 1994, p. 221 et suiv.)

93. Sandrine Pina, art. cité, p. 12.

94. Hans Kelsen, «La doctrine du droit naturel et le positivisme juridique », art. cité, p. 486.

95. Toujours en 1928, Kelsen soutient en effet que, "Si la fonction de la norme fondamentale hypothétique, comme cela a été montré précédemment, consiste à façonner le matériel juridique empirique en un ordre intelligible, c'est-à-dire dépourvu de contradictions, le résultat du positivisme critique semble alors se rapprocher de celui de la doctrine du droit naturel. Ce qui est essentiel, en l'occurrence, ce n'est pas le fait que tous les deux aboutissent à une justification du droit positif, le positivisme, de façon directe et consciente, et la doctrine du droit naturel, de façon indirecte et le plus souvent inconsciente. Mais c'est le fait que tous les efforts de la doctrine du droit naturel pour définir la mesure absolue de la [...] justice, débouchent finalement sur l'idée d'un ordre formel, c'est-à-dire un système dépourvu de contradictions, et donc sur une formule qui est en accord avec le droit positif en général» (ibid., p. 485-486).

96. Hans Kelsen, Das Problem der Souveränität und die Theorie des Völkerrechts, op. cit., p. 123, traduit et cité par Andràs Jakab, "Problèmes de la Stufenbaulehre », art. cité, p. 427. 
revêtu de ce caractère. Kelsen n'aurait fait ici qu'appliquer les principes de l'épistémologie néo-kantienne selon lesquels c'est la méthode qui produit son objet de connaissance ${ }^{97} "$.

\subsubsection{La science du droit, gardienne de l'unité logique de son objet}

Kelsen, dans un écrit de 1953, explicite fort bien comment l'unité se trouve associée au droit, par l'intermédiaire de la méthode normativiste $^{98}$ : «Cette unité [du droit] est logico-systématique et son critère négatif est l'absence de contradiction. Elle n'est pas immédiatement donnée dans la réalité juridique mais relève bien de la connaissance du droit. Le droit positif, une loi ou un traité international peuvent contenir des normes qui se contredisent mutuellement. Une loi peut révéler une contradiction par rapport à la Constitution ou au droit des gens [droit international]. Mais la connaissance du droit doit éliminer ces contradictions par le biais de l'interprétation et elle le fait toujours d'une manière ou d'une autre. Ainsi, c'est avant tout la connaissance scientifique qui permet au droit, comme à la nature, de devenir une totalité intelligible, c'est-à-dire dépourvue de contradictions ${ }^{99}$.»

Le projet de Kelsen semble ainsi moins correspondre à la description neutre de l'ordre juridique qu'à la restructuration cohérente et unitaire de ce dernier. L'on ne peut donc qu'être en désaccord avec N. Bobbio concernant l'objet de la métathéorie de Kelsen. Le juriste et philosophe turinois soulignait en effet que «La théorie de la science du droit de

97. Andràs Jakab, ibid.

98. C'est ce que Kelsen appelle dans sa Théorie pure du droit «L'unité logique de l'ordre juridique » : jusqu'à la $2^{\text {de }}$ éd. de la Théorie pure du droit (1960), Kelsen adopte une conception dite «hylétique » des normes. C'est-à-dire qu'il les considère comme des entités idéales, des devoir-être dépourvus de toute connexion avec le monde de l'être (contrairement à la conception dite "expressive» adoptée par les réalistes qui considèrent le droit comme un fait: le produit d'un acte d'interprétation d'un énoncé). Alors que, selon la conception expressive, une logique des normes n'apparaît pas possible, puisque n'est pas du domaine du possible une logique des comportements humains (et des phénomènes sociaux), Kelsen postule la possibilité d'une logique indirecte des normes, ce qui fonderait son unité. Indirecte car s'appliquant seulement médiatement aux normes, via les propositions descriptives émises par la science du droit. Il incomberait ainsi à la science juridique d'établir la logique du système juridique en recourant à diverses techniques permettant de résoudre les conflits de normes (voir Hans Kelsen, TPD, op. cit., p. 206-209).

99. Hans Kelsen, «Qu'est-ce que la théorie pure du droit ?», Droit et Société, $\mathrm{n}^{\circ}$ 22, 1992, p. 551-568, en particulier p. 556-557. 
Kelsen [sa métathéorie, dirait M. Troper ${ }^{100}$ ] "prescrit de décrire ${ }^{101 " ~ " . ~}$

100. Michel Troper, Pour une théorie juridique de l'État, op. cit., p. 33: «le positivisme est avant tout une métathéorie prescriptive. Selon la formule de Bobbio, il prescrit à la science de décrire. [...]. Telle est, en général, l'attitude de Kelsen ».

101. Norberto Bobbio, Essais de théorie du droit, op. cit., p. 191. 
Or, il apparaît que la science du droit doit, selon cette perspective métathéorique, décrire de façon à "dénaturer» l'objet d'étude, en le dotant d'une propriété qu'il n'avait pas sur la base de la seule connaissance : l'unité.

De même, l'on ne peut qu'exprimer un désaccord avec la conception de Michel Troper selon laquelle: «Le positivisme kelsénien est caractérisé par l'idée que la construction d'une science du droit est possible. Cela implique, par conséquent, une distinction entre le droit et la science du droit, une volonté de limiter cette science à la connaissance de son objet $^{102} \ldots »$

En effet, l'unité du droit n'est pas (ou pas totalement, du moins) issue de l'acte de connaissance du droit, mais d'un acte de volonté prescrit par Kelsen à la science du droit ${ }^{103}$. On peut même aller un peu plus loin en affirmant qu'en contradiction avec l'objet même de la science du droit de "poursuivre uniquement la connaissance du droit» et la description du droit tel qu'il est (c'est-à-dire la description d'un devoirêtre), la métathéorie kelsénienne aboutit à prescrire à la science du droit de décrire le droit tel qu'il devrait être... pour être conforme à l'image de la science telle que se la représente Kelsen ${ }^{104}$.

Il y a donc un présupposé axiologique à l'œuvre au cœur même de la Théorie pure du droit: le droit doit correspondre à l'objet d'une "véritable science ${ }^{105}$ ». La science n'est plus distincte de son objet puisqu'elle devrait - et, selon Kelsen, elle le fait en pratique ${ }^{106}$ - lui attribuer une qualité dont il n'est pas doté, ce, par un acte de volonté.

102. Michel Troper, "Le positivisme juridique", Revue de synthèse, Paris, Centre international de synthèse, philosophie et épistémologie juridiques, ${ }^{\text {os }} 118-119$, avril-septembre 1985 , p. 190.

103. Kelsen souligne dans sa Théorie pure $d u$ droit que «la connaissance du droit cherche $[. .$.$] à concevoir son objet comme un tout pleinement intelligible, et à le$ décrire en propositions non-contradictoires: par suite, elle part de l'idée que les conflits de normes peuvent être et doivent nécessairement être résolus [...] par la voie de l'interprétation» (Hans Kelsen, TPD, op. cit., p. 206-207).

104. Il s'agit, en 1934, d' « élever la science du droit [...] au niveau et rang d'une véritable science ( (ibid., préface de la $1^{\text {re }}$ éd., p. 3), en 1960, «de résoudre les problèmes les plus essentiels d'une théorie générale du droit d'après les principes de la pureté méthodologique de la science du droit, et de fixer, ce faisant, la position de cette science du droit au sein du système des sciences avec plus de précision que je ne

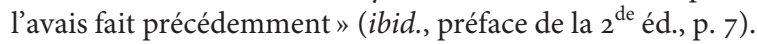

105. Ibid., préface de la $1^{\text {re }}$ éd., p. 3 .

106. Une pratique qui demeure, comme l'attestent les études approfondies de Philippe Jestaz et Christophe Jamin, (La Doctrine, recueil Dalloz, "Méthode du droit ", janvier 2004, 314 p. ; "L'entité doctrinale française », Paris, Dalloz, 1997, p. 167 et suiv.) 
Cette opération peut être perçue comme un acte additif, elle n'en reste pas moins une forme de mutilation de l'objet "brut» droit. Kelsen lui-même ne s'y trompe d'ailleurs pas qui énonce qu' «Avec le postulat d'un ordre intelligible, présentant un sens, c'est-à-dire dépourvu de contradictions, la science juridique dépasse déjà la limite du pur positivisme $^{107}$ ».

\subsubsection{Les implications de la métathéorie kelsénienne: une science du droit prescriptive}

En ce sens, les néo-normativistes semblent avoir quelques difficultés à appréhender les implications de la métathéorie kelsénienne. La science du droit qui se conforme à cette dernière ne peut qu'être prescriptive et affirmer sa préférence pour un système juridique ou un autre. Rappelons ici les propos d'Otto Pfersmann à propos de l'expression « concurrence des systèmes juridiques » : elle traduirait " un antagonisme politique au sens où les uns comme les autres [membres de la doctrine] désirent élargir la zone d'influence du système qui leur est cher et que les uns comme les autres craignent de voir se réduire l'attraction et l'influence de leur système préféré. [...] Les groupes antagonistes font par conséquent apparaître leur système préféré comme le meilleur et le système opposé comme truffé de défauts. Ils se comportent, sous cet angle, comme des acteurs économiques qui cherchent à vendre un produit ${ }^{108}$ ».

Ce point de vue, critique, ne permet pas d'entrevoir qu'en agissant comme des "acteurs économiques », les membres de la doctrine juridique $^{109}$ se conduisent conformément à la métathéorie kelsénienne. Car

107. Hans Kelsen, «La doctrine du droit naturel et le positivisme juridique », art. cité, p. 484 .

108. Otto Pfersmann, «Qu'entend-on exactement par l'expression «concurrence des systèmes juridiques », art. cité, p. 2595-2596.

109. La «doctrine" peut être présentée comme une catégorie juridique créée, de fait, par les juristes, jamais vraiment théorisée pour elle-même (Philippe Jestaz et Christophe Jamin, La Doctrine, op. cit., p. 1-8), et qui renvoie, comme le souligne Olivier Beaud ("Doctrine», dans Dictionnaire de la culture juridique, $o p$. cit., p. 385-388), principalement à deux sens non entièrement distincts. Celui d'un contenant: "l'ensemble des ouvrages juridiques, la littérature du droit » (p. 385); celui d'un contenu: "l'état des conceptions sur le droit et l'ensemble des solutions positives, telles que les reflètent les œuvres des écrivains juridiques » (Bonnecase, 1926, cité par Olivier Beaud, op. cit., p. 385). La définition que nous retiendrons ici correspond toutefois à une troisième acception, non entièrement distincte des deux précédentes: celle de "science du droit». Cette assimilation « doctrine»/«science du droit» a été contestée par Kelsen mais sa définition de l'objet de la science du droit aboutit précisément à faire de celle-ci une dogmatique 
de la même manière qu'il n'existe de conflits de normes qu'interprétés, il n'existe de concurrence entre les systèmes juridiques qu'interprétée comme telle. Le membre de la doctrine, qu'il soit constitutionnaliste ou " européiste » convaincu, au regard d'affirmations (jurisprudentielles) contradictoires de prévalence au sein de la Constitution française et de la jurisprudence des cours européennes ${ }^{110}$, applique à l'instar de Kelsen,

que lui-même ne juge pas, d'après ses propres critères, scientifique en ce qu'elle opère sur le droit des opérations de systématisation et de mise en cohérence. On admet donc ici: 1. qu'il est tout à fait possible de distinguer aujourd'hui entre science du droit et doctrine juridique, la première recherchant une position d'extériorité par rapport au droit, tandis que la seconde se placerait plus volontiers en position d'intériorité (voir Jacques Chevallier, «Doctrine juridique et science juridique », Droit et société, 50-2002, p. 103-119; Étienne Picard, «science du droit ou doctrine juridique ", dans L'Unité du droit, Mélanges Drago, Paris, Economica, 1996, p. 119 et suiv.; Sylvie Cinamonti, «Doctrine», dans André-Jean Arnaud, DETSD, op. cit., p. 186) ; 2. que la métathéorie kelsénienne, de par son ambiguité et l'exigence d'unité (comme projet) qui la sous-tend, tend toutefois à assimiler les deux concepts et les deux activités; 3 . que la distinction est relativement faible et ne peut être reconnue sur le simple fondement qu'à la science du droit correspondrait une activité scientifique tandis qu'à la doctrine correspondrait une activité dépourvue de scientificité et ce, pour les raisons suivantes: a. doctrine et science juridique sont partiellement intégrées institutionnellement; b. l'activité de chacune d'entre elles implique une part de l'autre; c. la science ne peut se définir que comme "une activité cognitive visant à donner une représentation du phénomène [étudié] conforme au paradigme scientifique adopté » (François Ost, art. cité, p. 540). Or, de fait, la doctrine «ne revendique pas l'extériorité de sa position comme un élément nécessaire d'un jugement scientifique. [...]. Elle ne partage pas la conception déterministe de la science [...] ou la conception épistémologique fondée sur la séparation rigide entre le matériau observé et l'observateur. Elle se contente d'une conception modeste de la science, au sens de "tout ensemble de connaissance raisonnées et coordonnées" » (Olivier Beaud, art. cité, p. 386). Où l’on voit que la doctrine adopte, conformément à la métathéorie kelsénienne, une épistémologie constructiviste. Le seul critère de distinction entre «science du droit » et « doctrine » pouvant être caractérisé par le degré d'interaction assumé entre le sujet et l'objet, à travers la définition d'un projet épistémologique distinct, selon l'une ou l'autre activité. Reste que cette distinction des perspectives de projet attend encore une mise en œuvre et, qu'en son absence, «l'hypertrophie de la fonction doctrinale transforme [...] les juristes en de simples artisans préposés au bon fonctionnement de l'ordre juridique (Jacques Chevallier, art. cité, p. 118-119). La distinction proposée par Jacques Chevallier entre doctrine juridique et science juridique apparaît, en ce sens, partiellement prescriptive.

110. Selon le Conseil constitutionnel, les traités (communautaires) bénéficient d'une autorité supérieure à celle de la loi en vertu de l'article L 55 C. 1958, et l'article L 88-1 C. implique une exigence constitutionnelle de transposition des directives européennes (CC, décision $\mathrm{n}^{\circ}$ 2004-496 DC du 10 juin 2004, considérant 7) dans l'ordre interne pouvant déroger à l'ordre interne, sauf à ce que ladite loi porte une atteinte à un principe inhérent à l'identité constitutionnelle de la France 
une position moniste. Reste alors à savoir à quel système sera attribuée la primauté. En l'absence d'unité réelle de systèmes en interrelations parfois conflictuelles, la «science du droit» (au sens où l'entend Kelsen) cherche à l'établir en affirmant la priorité d'un ensemble normatif sur un autre. Ainsi, de la même manière qu'elle devait résoudre, par son activité interprétative, les conflits de normes, selon Kelsen, se trouvet-elle devoir aujourd'hui résoudre les conflits systémiques (des normes de systèmes différents s'appliquant sur un même territoire et pour les mêmes situations, normes rentrant en conflits).

En outre, l'idée d'une "concurrence des systèmes juridiques " est parfaitement compréhensible et admissible si l'on admet les faiblesses de l'assimilation kelsénienne du droit et de l'État ${ }^{111}$ : comme le démontre habilement Michel Troper, la conception moniste de l'État et du droit repose sur le principe d'imputation qui aboutit à attribuer à l'État luimême (l'État lato sensu) les actes des organes juridiques de l'État (soit l'État stricto sensu). Étant donné que tout ordre juridique centralisé est un État (lato sensu), et que tout ordre juridique centralisé comporte inévitablement des organes juridiques spécialisés (État stricto sensu), le concept d'État lato sensu comporte inévitablement celui d'État stricto sensu et non l'inverse. Or, les organes juridiques peuvent se voir limités par le droit, et «ce sont précisément ce que la doctrine dualiste traditionnelle vise lorsqu'elle parle de l'État ${ }^{112}$ ". Parce que Kelsen reconnaît que le concept étroit (stricto sensu) d'État reste nécessaire, et que les concepts d' «État» comme celui de «système juridique » sont tous deux des concepts définis par le droit, alors les normes qui sont rapportés aux systèmes juridiques se rapportent en réalité aux actes de volonté des organes des dits systèmes. Dit autrement, l'expression doctrinale

(CC, décision $\mathrm{n}^{0}$ 2006-543 DC du 30 novembre 2006, considérant 6). Mais il affirme encore et toujours que la Constitution constitue «le point indépassable du sommet de la hiérarchie des normes en droit interne» (Louis Favoreu et al., Les Grandes Décisions du Conseil constitutionnel, $16^{\mathrm{e}}$ éd., Paris, Dalloz-Sirey, "Grandes décisions", septembre 2011, p. 387). La Cour de justice de l'Union européenne, quant à elle, affirmait dès 1964, la primauté du droit des traités européens sur les droits nationaux, y compris les constitutions nationales (CJCE, 15 juillet 1964, Costa c/. Enel, aff. 6/64, Recueil CJCE, p. 1160).

111. Cette quatrième marque importante de l'exigence d'unité de l'objet droit repose sur l'idée qu'il y a identité (dans le sens fort du contraire d'ipséité) du droit et de l'État; que les deux se confondent; que, par conséquent, la théorie de l'auto-limitation de l'État par le droit est une justification idéologique de l'autorité de l'État qui se trouve ainsi légitimée par le droit (voir Hans Kelsen, TPD, op. cit., p. 280-304; et TGDE, op. cit., p. 235-245).

112. Michel Troper, Pour une théorie juridique de l'État, op. cit., p. 154. 
« concurrence des systèmes juridiques» renvoie à la mise en concurrence institutionnelle d'ensembles normatifs par certains organes juridiques. Surtout, l'expression apparaît non seulement plausible mais encore parfaitement fondée en ce que les systèmes juridiques en concurrence avec l'ordre juridique de l'État sont, comme le démontre l'exemple de l'Union européenne, des ordres juridiques partiellement centralisés et dont la primauté dépend d'un critère organique: la présence au sein de l'ordre juridique interne (étatique) d'organes acceptant, sans y être contraints (de par l'absence de structures organiques centralisées pour contrôler cette application), d'appliquer le droit conventionnel. Ainsi, dans une conception moniste des rapports de système, il apparaît que lesdits organes, parce qu'ils sont communs à divers ordres juridiques, peuvent faire jouer un système contre l'autre, une norme suprême contre une autre. On retrouve l'idée selon laquelle il n'y a de concurrence normative qu'interprétée comme telle par un organe juridique. L'entrée en vigueur de la QPC en 2008 (sa mise en œuvre date de 2010), au moment même où $\mathrm{O}$. Pfersmann récuse l'expression «concurrence des systèmes juridiques » fournit pourtant bon nombre d'exemples. Nous n'en choisirons qu'un : celui des trois arrêts de la Cour de cassation en date du 15 avril $2011^{113}$. La juridiction suprême de l'ordre judiciaire français, censée ne pas censurer les articles litigieux du Code de procédure pénale relatifs à la garde à vue eu égard à la décision n ${ }^{0} 14 / 22$ QPC $\mathrm{du}$ Conseil constitutionnel ${ }^{114}$ qui intimait aux juridictions ordinaires de ne prendre aucune mesure relative aux dits articles, dans l'attente de la nouvelle loi devant modifier l'ancienne jugée inconstitutionnelle, et eu égard surtout à l'autorité des décisions du juge constitutionnel français, écartait pourtant les articles incriminés en se prévalant de l'autorité interprétative des arrêts de la Cour européenne des droits de l'homme (Cour EDH). La concurrence, ici orchestrée par un organe étatique, l'est entre deux normes suprêmes (régissant deux systèmes distincts) telles qu'interprétées par leurs juges " authentiques " : la Constitution française de 1958 et la convention de sauvegarde des droits de l'homme et des libertés fondamentales du 4 novembre 1950.

Pour Otto Pfersmann, puisqu'il apparaît impossible de résoudre la concurrence entre certains systèmes juridiques en se fondant sur le droit existant, la dogmatique «ne produira autre chose que de l'idéologie ${ }^{115}$ ».

113. Cass., ass. plén., 15 avril 2011, $\mathrm{n}^{\circ}$ 10-17.049, Recueil Dalloz, 2011. p. 1080.

114. CC, décision $n^{0} 14 / 22$ QPC du 30 juillet 2010.

115. Otto Pfersmann, «Qu'entend-on exactement par l'expression «concurrence des systèmes juridiques», art. cité, p. 2598. 
Mais il apparaît que les conflits de normes ne peuvent non plus être résolus par la doctrine, comme l'affirme pourtant Kelsen. En effet, si l'on admet, à l'instar de Kelsen, que le juge se livre à une activité interprétative fondée sur un acte double, cognitif et volitif ${ }^{116}$, la doctrine ne peut, à l'avance, prévoir ce que le juge dira afin de résoudre lesdits conflits (justification), ni quelle norme il fera prévaloir (effectivement). Kelsen recommande alors de se référer à des principes généraux de résolution des conflits ${ }^{117}$, mais il n'existe aucun système objectif de règlement des conflits de normes et, quand bien même il en existerait un, le caractère - en dernier ressort - volitif de l'interprétation du juge et les circonstances de l'espèce ne permettraient pas d'en faire un référent absolu et certain ${ }^{118}$, mais plutôt évolutif et toujours conditionné à des

116. Pour Hans Kelsen, «dans l'application du droit par un organe juridique, l'interprétation du droit à appliquer, par une opération de connaissance, s'unit à un acte de volonté par lequel l'organe créateur de droit fait un choix entre les possibilités révélées par l'interprétation à base de connaissance. Ou bien cet acte crée une norme de degré inférieur, ou bien il réalise l'acte de contrainte ordonné dans la norme juridique à appliquer» (Hans Kelsen, TPD, op. cit., p. 340).

117. Pour des normes générales posées par un seul et même organe ou par deux organes différents, mais à des moments différents (ibid., p. 207).

118. L'on songe ici notamment à la jurisprudence de la Cour de cassation relative aux rapports de systèmes. Ainsi, en 2007, la Cour de cassation mentionnait dans les visas de ses arrêts, étape importante et imprévisible, la jurisprudence de la Cour européenne des droits de l'homme (Soc. 13 juin 2007, pourvoi $n^{0}$ 05-45.694, Recueil Dalloz, 2007, p. 2439, note Cécile Pérès; et notamment le commentaire de la décision par Pascale Deumier, «L'avènement des arrêts de la Cour européenne des droits de l'homme au visa des arrêts de la Cour de cassation ", RTD Civ., 2007, p. 536: toute cour évoluant dans un système juridique au sein duquel l'absence de force du précédent ne permet pas d'assurer l'uniformité des décisions jurisprudentielles, légitime ses arrêts en insistant sur la réception de fait des autres juridictions. Un processus que la Cour de cassation connaît parfaitement puisqu'elle affirmait en 1980 que chaque juridiction inférieure de l'ordre judiciaire doit «faire (sienne) la doctrine énoncée dans un arrêt de la Cour de cassation » (Soc. 29 avril 1980, bull. civ. v, $\mathrm{n}^{\circ}$ 371). «Or, cette démarche implique [dans le cadre des relations entre Cour EDH et Cour de cassation] l'appropriation de la règle portée et non la reconnaissance de l'autorité formelle de sa source, à l'inverse de ce que traduit l'insertion de l'arrêt [de la Cour EDH] au visa [de l'arrêt de la Cour de cassation]. Par cette présence au fondement juridique de la cassation, la chambre sociale reconnaît à la décision européenne une autorité de droit et l'élève à la force du précédent. Une telle reconnaissance est étonnante [car elle] n'est nulle part exigée, ni dans le système interne, ni dans le système européen [...] ».). Pascale Deumier voit dans cette référence explicite à la jurisprudence de la Cour EDH une "soumission à une autre autorité morale [que celle de l'Assemblée plénière de la Cour de cassation], celle qui a le pouvoir du dernier mot", ainsi qu'une "solution réaliste» consistant à s'abriter derrière la jurisprudence de la Cour européenne lorsque se trouve mise en cause la législation nationale. 
considérations de politique juridique ${ }^{119}$ (que l'on peut décrire mais en aucun cas anticiper de façon certaine).

Ainsi, Kelsen lui-même se trouve contraint d'affirmer qu'en cas de conflit entre deux normes posées en même temps par un même organe, deux possibilités s'ouvrent:

- «ou bien on peut interpréter les deux dispositions en ce sens que les organes chargés d'appliquer la loi, les tribunaux par exemple, se voient reconnaitre le pouvoir de choisir entre les deux normes;

- ou bien [...] on peut admettre que l'une des deux normes limite la validité de l'autre ${ }^{120}$.»

Il appert donc que :

- dans le premier cas, le conflit n'est pas résolu et les normes ne peuvent être interprétées par la science du droit sans contradictions;

- dans le second, il revient à la science du droit d'opérer un choix, en faisant primer l'une des deux normes sur l'autre mais, chacun pouvant opérer un choix différent selon son interprétation propre, les propositions descriptives de la science du droit seront amenées à rentrer elles-mêmes en contradiction.

Enfin, n'oublions pas que s'offre à la science du droit un choix entre ces deux alternatives et que ce choix lui-même ouvre la perspective d'une contradiction des propositions de la science du droit.

119. Voir la note précédente. On peut, en outre, se reporter aux trois arrêts du 15 avril de la Cour de cassation par lesquels, bafouant l'autorité de la chose jugée par le Conseil constitutionnel en vertu de l'articleL 62 C. 1958, elle reconnaissait l'autorité interprétative de la jurisprudence de la Cour européenne des droits de l'homme (Cass., ass. plén., 15 avr. 2011, $\mathrm{n}^{\mathrm{o}}$ 10-17.049, Recueil Dalloz, 2011, p. 1080). Par ces trois arrêts, la Cour de cassation fait en effet jouer, contre la solution juridictionnelle du Conseil constitutionnel, l'inconventionnalité des dispositions contestées. Il y a avait, auparavant, conflit de normes entre les jurisprudences constitutionnelles et conventionnelles (Cour européenne des droits de l'homme) concernant la garde à vue. Un conflit que le juge constitutionnel avait tranché de façon à le résoudre en donnant au législateur français 11 mois pour modifier sa législation. La Cour de cassation a, de nouveau, le 15 avril, instauré un conflit de normes... pour des raisons de politique juridique, «S'inscrivant dans un contexte de crise larvée, que l'on arrive à supputer même depuis Limoges" (Jean-Pierre Marguénaud, «La reconnaissance par l'Assemblée plénière de la Cour de cassation de l'autorité interprétative des arrêts de la Cour européenne des droits de l'homme ou : la révolution du 15 avril », RTD Civ., 2011, p. 725-732). L'on relèvera en outre que le commentaire cité est celui d'un auteur favorable à ladite jurisprudence de la Cour de cassation.

120. Hans Kelsen, TPD, op. cit., p. 207. 
On pourrait alors prétendre, comme l'affirme O. Pfersmann, que la science du droit opère un choix en fonction d'une idéologie. Mais il s'agit là d'une conséquence nécessaire et inévitable de la métathéorie kelsénienne, et non d'un de ses effets indésirables.

En outre, Kelsen met, concernant la présentation par la science du droit d'un ordre dépourvu de contradictions, son propre raisonnement en défaut: il admet en effet qu'en cas de "conflit entre deux normes individuelles, par exemple deux décisions de justice [...]. Le conflit est résolu par le fait que l'organe chargé de l'exécution matérielle des normes a le choix de se conformer ou à l'une ou à l'autre des deux décisions ${ }^{121}$...» Or, si l'interprétation scientifique, qui n'est censée être que pur acte de connaissance ${ }^{122}$, reconnaît le conflit, elle ne peut le résoudre; il appartient à l'organe qui applique les décisions de justice de le trancher par un acte de volonté.

Soit donc l'on admet que qu'il est impossible à la science du droit de décrire le droit comme un tout unitaire "pleinement intelligible», soit l'on admet qu'elle ne peut le faire autrement qu'en ajoutant à l'acte de connaissance de l'interprétation scientifique un acte de volonté. Puisque l'unité du droit est, aux yeux de Kelsen, tant la condition de sa pureté que celle de son étude, il faut se résoudre à accepter que la science du droit, se conformant à la métathéorie kelsénienne, se fera nécessairement prescriptive.

Ainsi, confrontés à des réalités juridiques et institutionnelles différentes, normativistes et néo-normativistes prescrivent tous deux à la science juridique de résoudre les conflits et concurrences «dans le cadre des matériaux normatifs qui lui sont donnés - ou plus exactement imposés ${ }^{123} »$. Mais aucun des deux ne semble accepter l'inévitable conséquence de cette exigence d'unité logique de la représentation scientifique. Reste que la dogmatique constitue bien, conformément aux vœux de Kelsen, un élément favorisant la clôture normative du système juridique, à la fois en en donnant une représentation systématique et ordonnée, et en proposant aux organes juridiques, par cette activité interprétative sytématisante, des solutions normatives concourant à faire

121. Ibid., p. 208.

122. En effet, pour Hans Kelsen, la distinction entre l'interprétation des organes juridiques et l'interprétation scientifique (celle opérée par la science du droit) réside dans le fait que, tandis que la première est un acte double, de connaissance et de volonté, la seconde n'est qu'un acte de connaissance (ibid., p. 341-342).

123. Ibid., p. 206-207. 
de ces systèmes des "systèmes juridiques fermés ${ }^{124}$ ", du moins dans les pays où elle existe. L'activité dogmatique de la science du droit kelsénienne apparaît ainsi comme une science pratique, impliquant un travail de recherche, d'interprétation, de systématisation, et de proposition. Si « idéologie» il y a au fondement de cette activité, alors c'est celle-là même que préconisait Kelsen : celle de l'unité et de la cohérence.

La coexistence, au sein de la doctrine de Hans Kelsen, d'une théorie (description de la structure de l'ordre juridique) et d'une métathéorie (prescription à la science du droit de décrire le droit tel qu'il est) amène nécessairement une question supplémentaire: si la métathéorie de Kelsen est prescriptive (nous avons vu qu'elle prescrit à la science du droit de décrire mais également d'ordonner le droit), sa théorie peut-elle être seulement descriptive? Comme le souligne M. Troper, «ces deux aspects [théorie et métathéorie] ne sont pas entièrement séparés [et] Kelsen s'est efforcé, dans sa théorie du droit, d'appliquer ses propres prescriptions ${ }^{125}$ ». Dit autrement, au vu de nos conclusions quant aux prescriptions kelséniennes adressées à la science du droit, il nous faut nous demander si Kelsen n'appose pas lui-même sur l'objet droit une unité, afin d'élever celui-ci au statut de véritable objet scientifique.

\subsection{L'objet de la théorie pure du droit : bâtir une structure juridique unitaire}

Cette exigence d'une représentation unitaire du droit est également perceptible au sein de la théorie du droit de Kelsen, à trois niveaux différents au moins :

- l'unité de l'interprétation du droit (2.2.1);

- l'unité structurelle du droit, ou la prescription de ce que devrait être le droit (2.2.2);

- l'unité par la « légitimité », concept kelsénien à géométrie variable (2.2.3).

\subsubsection{L'unité de l'interprétation juridique:}

la science du droit comme unique source de droit?

D'une part, le matériau juridique, contrairement aux objets des autres sciences, produirait parfois une auto-interprétation " qui anticipe et

124. René David et Camille Jauffret-Spinosi, Les Grands Systèmes de droit contemporains, 11 e éd., Paris, Dalloz, «Précis», p. 267-296.

125. Michel Troper, Pour une théorie juridique de l'État, op. cit., p. 45. 
empiète sur l'interprétation que la connaissance juridique a mission de donner ${ }^{126} »$.

Pourtant, si, pour reprendre un exemple de Kelsen, des hommes réunis pour constituer un Parlement déclarent qu'en faisant telle chose, ils votent ou décident une loi, et si cette auto-interprétation se retrouve dans le contenu de la norme, alors ce contenu spécifique est un devoirêtre. Et, si la science du droit a pour objet le droit tel qu'il est, comme le suggère Kelsen, et non le droit tel qu'il devrait être, alors il ne peut y avoir d'empiètement du droit sur la science du droit (et réciproquement) puisque les deux sont distincts. Ainsi, si la norme édicte qu'elle est une loi, alors il s'agit inévitablement d'un devoir-être qu'il faut rendre de la manière suivante : "cette norme doit être considérée comme une loi. »

À moins, et c'est ce que suggère ici Kelsen, qu'il appartienne à la science du droit de dire ce qui constitue du droit ou pas ${ }^{127}$. Or, Kelsen

126. Hans Kelsen, TPD, op. cit., p. 11.

127. On retrouve cette idée fondamentale dès les premières pages de la Théorie pure $d u$ droit : «Seulement, en tant qu'évènements, c'est-à-dire considérés comme des éléments du système nature, ils ne sont pas objet d'une connaissance spécifiquement juridique et, par suite, ne sont en aucune manière quelque chose de juridique » (TPD, op. cit., p. 4). Michel Troper « observe ici que pour Kelsen "être objet d'une connaissance spécifiquement juridique" est l'équivalent d'être quelque chose de juridique" " (Michel Troper, Pour une théorie juridique de l'État, op. cit., p. 61). Pourtant, le "par suite» introduit entre les deux expressions de Kelsen démontre qu'il n'y a pas identité de sens entre elles, mais succession (chrono)logique: c'est parce que l'objet peut faire l'objet d'une connaissance spécifiquement juridique qu'il est quelque chose de juridique. Toutefois, il n'existe pas de "connaissance spécifiquement juridique ", il n'existe qu'une interprétation qui confère à l'objet d'une connaissance le caractère de « juridique». Partant, si c'est à la suite de cette activité d'interprétation que "quelque chose est juridique", alors c'est bien la science du droit qui décide ce qui est du droit et ce qui n'en est pas. Le positivisme kelsénien s'apparente donc bien à un constructivisme. Le projet apparaît d'ailleurs dans l'adverbe «spécifiquement ». En effet, il ressort également de l'activité d'interprétation (et non de connaissance, comme l'affirme Kelsen) que l'objet est qualifié de «spécifiquement» juridique. C'est donc l'interprétation qui confère son unité et sa pureté à l'objet et non l'objet qui est spécifiquement juridique, avant interprétation. Il s'agit bien d'une visée prescriptive qui se répercute sur la science juridique (qui ne doit connaître que son objet) mais également sur l'objet lui-même que l'on débarrasse de tout lien (ou connexion) avec un élément défini comme étranger. On retrouve cette double répercussion (induite par le néo-kantisme de Kelsen) lorsque Kelsen explique au lecteur ce que signifie «La pureté » : Pourquoi se dénomme-t-elle elle-même une théorie "pure" du droit? C'est pour marquer qu'elle souhaiterait simplement assurer une connaissance du droit, du seul droit, en excluant de cette connaissance tout ce qui ne se rattache pas à l'exacte notion de cet objet. En d'autres termes, elle voudrait débarrasser la science du droit de tous les éléments qui lui sont étrangers. Tel est son principe méthodologique fondamental» 
affirme que le droit s'autorégule et que la science du droit est distincte de son objet. Le chercheur en sciences juridiques n'est donc censé avoir aucune compétence pour affirmer ce qui doit être considéré comme droit ou pas. Il ne peut que prendre acte du devoir-être existant et non lui dénier existence (ou dénier l'existence d'une partie de ce devoir-être).

Il faut donc admettre, au vu de ce paradoxe de la pensée kelsénienne, que les normes juridiques ne sont pas données, mais bien, comme nous l'affirmons, (re)construites. Là même où Kelsen affirme l'unité spécifique du droit en tant qu'objet - qui serait le seul à produire une forme d'auto-interprétation ${ }^{128}$ - se fait jour une interaction objet/observateur scientifique.

(Hans Kelsen, TPD, op. cit., p. 9). Là, par contre, Kelsen établit une relation d'équivalence à travers l'expression «en d'autres termes ", alors même que les deux propositions n'ont pas la même signification. D’une part est affirmée la volonté de ne connaître que le seul droit; d'autre part, celle de débarrasser la science du droit de considérations politiques, religieuses, morales, etc. Il apparaît déjà, dès cette introduction, que la science du droit n'est pas complètement distincte de son objet puisqu'il y a interaction nécessaire entre la science et l'objet.

128. Ce qui nous paraît erroné, au regard de la situation de l'historien étudiant une quelconque révolution au cours de laquelle des écrits révolutionnaires n'ont de cesse de commenter les évènements comme correspondant à une "révolution »... Que l’on songe également au médecin confronté au patient qui lui énonce: " je suis malade». Historiens et médecins considèrent-ils alors ces actes autointerprétatifs comme une forme d'empiétement sur leur activité scientifique, ou plutôt comme une indication à prendre en compte (sans pour autant l'accepter d'emblée comme véridique) ? La difficulté, s'agissant du droit, est que, selon Kelsen, il s'autoproduit et la science du droit ne peut que le connaître et non l'énoncer. Tandis que c'est l'historien qui définit s'il a affaire à une révolution et le médecin qui conclut s'il a bien affaire à un malade. L'on peut alors aborder le problème de la manière suivante: les Grecs de l'Antiquité attribuaient leurs victoires militaires à la volonté des Dieux. L'historien (agnostique, ou monothéiste) repousse-t-il cette auto-interprétation comme un empiétement obscurcissant l'histoire (son champ d'étude que lui-même définit), ou comme un élément constitutif de l'histoire sans lequel on ne peut comprendre la civilisation grecque antique? La réponse paraît évidente. Et l'on voit pointer l'imperfection kelsénienne liée à sa méthode: 1. l'objet droit n'est pas connu mais bien construit. 2. La construction est toutefois élaborée à partir d'une délimitation préalable réductrice, ne permettant pas de prendre en compte ce que les organes juridiques, seuls créateurs de droit selon Kelsen, estiment être du droit. D’un point de vue épistémologique, il apparaît alors que ce n'est pas, comme le prétend Kelsen, le droit qui, par son auto-interprétation, empiéterait sur sa connaissance, mais bien le scientifique qui, par son activité de connaissance, interfère avec son « objet ». 


\subsubsection{L'unité structurelle du droit: ce que le droit devrait être}

D'autre part, et cela rejoint ce qui vient d'être dit, Kelsen affirme l'existence de normes "primaires" (prescrivant une sanction) et de normes "secondaires " (prescrivant une obligation). Il appartiendrait à la science du droit de distinguer ces normes afin de mieux les réunir au sein des propositions de la science du droit.

Cette description de la structure de l'ordre juridique peut se comprendre et apparaît, de fait, fort utile dès qu'il s'agit de déterminer, en cas de non-respect d'une obligation, la sanction correspondante: "Si l'on dit que l'obligation juridique "doit" être satisfaite, ce "doit" est, d'une certaine façon, un "épiphénomène" du droit de la sanction. Cette formulation présuppose que la norme juridique se divise en deux normes distinctes, deux énoncés de "devoir-être" : un individu déterminé "doit" observer une certaine conduite, et un second individu "doit" exécuter la sanction en cas d'infraction à la première norme. [...] Si la première norme, qui proscrit le vol, est supposée valide à condition que la seconde norme attache une sanction au vol, alors dans un exposé exact du droit, la première norme s'avère superflue. La première norme, si tant est qu'elle existe, se trouve contenue dans la seconde, la seule véritable norme juridique $^{129}$.»

Kelsen admet toutefois que "si nous admettons l'existence de la première norme [c'est-à-dire l'obligation], la représentation du droit s'en trouvera grandement facilitée ${ }^{130}$ », mais souligne «qu'un tel procédé ne se justifie que si nous gardons à l'esprit que la seule norme juridique véritable est une norme pourvue d'une sanction ${ }^{131}$ ".

La science du droit devrait ainsi déterminer et distinguer les normes juridiques véritables, celles pourvues de sanctions, des autres, non véritablement juridiques. Cette conception apparaît déformante pour la structure même de l'ordre juridique pour au moins quatre raisons :

1. toutes les normes n'édictent pas des sanctions et ne se rattachent pas forcément à une sanction ${ }^{132}$;

129. Hans Kelsen, TGDE, op. cit., p. 111.

130. Ibid.

131. Hans Kelsen, TGDE, op. cit., p. 114.

132. Tel est le cas de la plupart des dispositions du droit constitutionnel et du droit international «au point [comme le rappelle Paul Amselek] qu'on a pu parfois mettre en doute la juridicité de ces deux branches du droit!» (Paul Amselek, Cheminement philosophiques dans le monde du droit et des règles en général, Paris, Armand Colin, «Le temps des idées", octobre 2012, p. 236). 
2. il n'est pas évident (au contraire) qu'une seule et même norme regroupe à la fois l'obligation et la sanction ${ }^{133}$;

3. dans le cas de normes distinctes, la norme édictant l'obligation devrait logiquement, dans ce cas, être édictée en premier ;

4. plus la sanction est lourde, plus l'on peut estimer que l'obligation à respecter est jugée importante au sein d'une société.

Selon un rapport de causalité il apparaît donc que, si l'obligation est jugée importante par les organes du système juridique, ils y attacheront (au sein de la même norme, ou au sein d'une autre norme) une sanction d'autant plus grave. La norme qui détermine la gravité de la sanction est donc celle qui pourrait être qualifiée de primaire sous un double rapport, à la fois logique et causal.

Dans tous les cas, il n'apparaît pas que l'on puisse dénier le caractère de norme juridique véritable à une norme, primaire ou secondaire, si le système juridique auquel cette norme appartient ne le fait pas. À moins d'adopter une représentation prescriptive du droit.

On pourrait se contenter de voir dans la distinction normes primaires/normes secondaires ${ }^{134}$, une marque de l'exigence d'unité, comme le fait $\mathrm{H}$. Hart ${ }^{135}$. Mais cette critique, fondée, apparaît limitée en ce

133. Comme le souligne Paul Amselek, « un survol rapide des dispositions que l'on trouve dans les codes juridiques fait apparaître qu'elles ne sont pas toutes, ni même pour la plupart d'entre elles, construites sur le moule logique "si...alors" (ibid., p. 244). On peut, au titre des raisons 1 et 2 - et avec Paul Amselek -, évoquer les articles 516 à $536 \mathrm{du}$ Code civil concernant la distinction (et définition) des biens ainsi que, surtout, l'article 371 du même Code : «L'enfant, à tout âge, doit honneur et respect à ses père et mère. »

134. Cette distinction entre normes aux fins d'une meilleure représentation (unitaire) du droit se retrouve chez les néo-normativistes. Voir notamment Otto Pfersmann, "Temporalité et conditionnalité des systèmes juridiques", art. cité, p. 222-245. L'auteur évoque une temporalité interne aux systèmes juridiques, chaque norme du système comportant une "double détermination temporelle: un domaine conditionnel («si...»), et un domaine conséquentiel («alors...»)»

135. "L'unité appréciable du modèle auquel [ces théories] réduisent toutes les règles de droit [a pour conséquence une] déformation des différentes fonctions sociales que remplissent ces différents types de règles de droit ». Ainsi, pour Herbert Hart, «nous dissimulerions le mode caractéristique de fonctionnement [des règles édictant l'obligation d'une conduite], en nous limitant aux règles qui enjoignent aux tribunaux d'infliger des sanctions en cas de désobéissance, ou en les faisant apparaître comme primaires, étant donné que ces dernières règles pourvoient à l'insuccès ou à l'échec du but premier assigné au système. Elles peuvent, il est vrai, s'avérer indispensables, mais elles ne remplissent qu'une fonction auxiliaire. L'idée selon laquelle les règles substantielles du droit pénal ont pour fonction (et, en un sens large, pour signification) de régir non seulement l'activité des 
qu'elle ne rend pas compte de l'objet premier de cette "reconstruction " kelsénienne de la structure de l'ordre juridique. Il ne s'agit pas simplement d'une prescription adressée à la science du droit afin de faciliter la représentation du droit en cherchant à associer, à toute obligation, la sanction qui correspond à son non-respect. La science du droit doit également être plus exigeante au respect de la norme, non par les sujets de droit destinataires de l'obligation, mais par les organes juridiques, c'est-à-dire ceux qui appliquent le droit.

Comme le souligne Kelsen, «Si nous admettons l'expression courante selon laquelle le sujet obéit ou désobéit au droit, nous admettrons l'énoncé : l'organe "applique" ou n" "applique" pas le droit. Seule cette distinction terminologique nous permettra de saisir avec netteté la différence entre le rapport du droit au sujet, délinquant potentiel, et le rapport du droit à l'organe. Dans la mesure où, par droit, nous entendons une véritable norme juridique primaire, le droit est efficace s'il est appliqué par l'organe - si l'organe exécute la sanction. [...] Ainsi, bien que l'efficacité du droit signifie avant tout que le droit est appliqué par l'organe approprié, elle signifie en second lieu que les sujets obéissent [globalement] au droit ${ }^{136}$ ».

En effet, que cent individus n'obéissent pas à la loi n'entraîne pas la remise en cause du système juridique. Mais que dix organes juridiques n'appliquent pas les sanctions qu'ils ont la charge de mettre en œuvre, et se trouvent mises en péril tant l'efficacité du système de sanctions que celle du système d'obligation. La science du droit a donc pour vocation, selon Kelsen, à travers une certaine déformation de la structure même du droit, à mettre en lumière l'application effective du droit par les organes juridiques, à souligner que c'est celle-ci qui est primaire. Car de celle-ci dépend l'efficacité de l'ordre juridique. Ainsi, en mettant en exergue les normes prévoyant des sanctions et en les considérant comme seules normes véritables, la science du droit contribue à prescrire une certaine efficacité au droit. Car l'objet «droit», pour le rester, doit être efficace.

On pourrait être tenté d'affirmer que Kelsen ne fait que décrire un état de fait. Le seul vrai droit est le droit globalement et généralement efficace.

autorités dans l'application d'un système de peines, mais encore l'activité des simples citoyens dans leur vie privée, ne peut être éliminée sans jeter par-dessus bord des distinctions essentielles et sans obscurcir le caractère spécifique du droit comme un moyen de contrôle social (H. L. A. Hart, Le Concept de droit, trad. Michel Van de Kerchove, postface Penelope A. Bulloch et Joseph Raz, $2^{\mathrm{e}}$ éd. revue et augmentée (éd. originale, 1961), Publication des facultés universitaires Saint-Louis, Bruxelles, janvier 2006, p. 57-58 [p. 38-39 pour l'éd. originale]).

136. Hans Kelsen, TGDE, op. cit., p. 112. 
Mais, parce qu'il prescrit une distinction terminologique spécifique, il démontre que la science juridique ne doit pas se contenter de décrire le droit tel qu'il est; elle doit également veiller à n'étudier que du droit, c'est-à-dire du droit efficace. La critique de Hart tombe alors d'elle-même puisque c'est parce que Kelsen sait trop bien que c'est le système d'obligations qui est premier, qu'il convient de le préserver en mettant l'accent sur le système de sanctions et les organes chargés de les appliquer. Reste que, de cette manière, Kelsen ne dit pas que l'obligation juridique dépourvue de sanctions n'est pas du droit selon l'ordre juridique considéré, mais bien ce que lui-même, Hans Kelsen, considère ne pas en être. On se trouve donc à nouveau devant une prescription de ce que le droit devrait être: "Une norme juridique est une norme primaire, qui prescrit une sanction ${ }^{137}$.»

\subsubsection{L'unité par la « légitimité», concept kelsénien à géométrie variable}

Nous avons déjà noté que la validité correspond, chez Kelsen, à un rapport de production (ou «rapport selon l'ordre de la production»). C'est en effet l'appartenance à un système de normes, à travers ce rapport de dépendance entre les normes, qui permet de les qualifier de

137. Ibid. D'ailleurs, le désaccord entre Kelsen et Hart sur la distinction entre règles primaires (obligations pour Hart, sanctions pour Kelsen) et secondaires (relatives aux règles primaires pour Hart; obligations pour Kelsen), ne peut qu'être relativisé au regard de l'affirmation hartienne de ce qui différencie le monde pré-juridique du monde juridique: «il est évidemment possible d'imaginer une société dépourvue de législateur, de tribunaux ou d'autorités publiques de toute espèce. [...]. le seul moyen de contrôle social [y] réside dans cette attitude générale du groupe à l'égard de ses propres modèles de comportement, en fonction de laquelle nous avons caractérisé les règles d'obligation. [...]. une telle structure sociale [...] est une structure de règles primaires d'obligations » (H. L. A. Hart, Le Concept de droit, op. cit., p. 110-111 [91]. Ce monde pré-juridique passerait au stade proprement juridique par l'adoption de "règles secondaires [qui] fournissent les "sanctions" publiques et centralisées du système» (ibid., p. 117 [98]). Le droit ne peut ainsi être conçu, selon Hart, que comme «l'union de règles primaires et secondaires" (ibid., p. 99 [79]). Il paraît alors tentant, puisque les normes établissant une sanction sont caractéristiques du passage du non droit au droit, de décrire comme juridiques uniquement ces normes ci, comme le fait Kelsen. Mais on ne pourrait le faire qu'en opérant une découpe à l'intérieur de l'objet droit, sur des critères qui ne sont pas donnés, mais bien interprétés comme déterminants, et à l'encontre même du langage que constitue le droit et qui qualifie de juridique les règles d'obligation, quand bien même elles seraient distinctes des règles établissant la sanction devant garantir son observance. 
proprement juridiques ${ }^{138}$. Ainsi, dans un premier temps, Kelsen affirme que «Le principe qu'une norme ne peut être posée que par l'organe compétent, c'est-à-dire par l'organe habilité à cet effet par une norme supérieure, est le principe de légitimité ${ }^{139}$ ». Une norme légitime, au sens ci-dessus défini, est donc valide. Dit autrement, la légitimité est une condition de la validité.

Mais Kelsen affirme autre part que le principe de légitimité est celui consistant en ce que "les normes d'un ordre juridique valent aussi longtemps que leur validité ne prend pas fin d'une façon qui est déterminée par cet ordre juridique, ou qu'elle ne fait pas place à la validité d'une autre norme de cet ordre ${ }^{140}$ ».

On se retrouve alors avec une signification inversée : la validité est une condition de la légitimité.

En outre, selon Kelsen, «ce principe [de légitimité] ne vaut que sous certaines conditions. Il n'est plus [opérant] dans le cas d'une révolution, prise au sens le plus large du mot, de façon à englober ce que l'on appelle un coup d'État. [...] Les individus hissés au faîte du pouvoir par la révolution se contentent habituellement d'annuler la Constitution et quelques lois revêtant une certaine importance politique, qu'ils remplacent par d'autres normes. L'ordre juridique précédent "demeure" en grande partie valide dans le cadre de l'ordre nouveau. Toutefois, l'expression "demeure valide" ne fournit pas de description appropriée

138. "Les systèmes de normes qui se présentent comme des ordres juridiques ont pour l'essentiel un caractère dynamique. Une norme juridique n'est pas valable parce qu'elle a un certain contenu, c'est-à-dire parce que son contenu peut être déduit par voie de raisonnement logique, d'une norme fondamentale supposée, elle est valable parce qu'elle a été créée d'une certaine façon, et plus précisément, en dernière analyse, d'une façon qui est déterminée par une norme fondamentale, norme supposée; c'est pour cette raison, et pour cette raison seulement qu'elle fait partie de l'ordre juridique dont les normes sont créées conformément à cette norme fondamentale» (Hans Kelsen, TPD, op. cit., p. 197). C'est parce que le critère d'appartenance au système juridique est quasi-exclusivement formel que, pour Kelsen, les ordres juridiques peuvent être qualifiés de dynamiques. Un système purement «statique» serait celui dans lequel le rapport entre les normes ne serait pas un rapport de production, mais un rapport de conformité. Dans un ordre statique, c'est en fonction de leur contenu, aisément subsumé sous celui de la norme supérieure, que les normes trouveraient le fondement de leur validité.

139. Ibid., p. 272.

140. Ibid., p. 209. Voir également Hans Kelsen, TGDE, op. cit., p. 171: «La validité des normes peut être limitée dans le temps, et il convient de noter que le terme et le début de cette validité sont exclusivement déterminés par l'ordre auquel ces normes appartiennent. Elles demeurent valides tant qu'elles ne perdent pas leur validité selon le procédé déterminé par l'ordre juridique. Tel est le principe de légitimité.» 
du phénomène. Seul le contenu des normes demeure inchangé, et non le fondement de validité. [L'ancienne Constitution] a été remplacée par une nouvelle [...]. Nul juriste ne saurait prétendre qu'après une révolution victorieuse l'ancienne constitution et les lois fondées sur celleci restent en vigueur, sous prétexte qu'elles n'ont pas été abrogées selon le procédé prévu par l'ordre précédent ${ }^{141} »$.

Le construit kelsénien apparaît alors relativement confus.

Si l'on respecte la loi de Hume, l'on ne peut inférer de la Révolution (fait ou Sein), ni la fin de validité des anciennes normes, ni l'entrée en vigueur des nouvelles (devoir-être ou Sollen). Kelsen, contraint de trouver une explication juridique pour le passage du droit au fait (et du fait au droit), fait alors émerger le concept de «légitimité», dans le second sens que nous avons déterminé.

Le juriste n'étudiant que le droit (Sollen) ne peut mêler l'être et le devoir-être. Il est donc normalement conduit à ignorer la Révolution en cours et à continuer de prétendre que les normes non abrogées selon la procédure prévue sont valides... sauf, comme tente de le faire accepter Kelsen, à considérer (enfin) la relation du fait au droit en recourant au «principe de légitimité » qui caractérise à la fois les normes (qui appartiennent au monde du devoir-être) et les organes juridiques (qui appartiennent au monde de l'être): «Une révolution se produit dès que l'ordre juridique d'une communauté est annulé et remplacé par un nouvel ordre de façon légitime, c'est-à-dire d'une façon qui ne soit pas prescrite par le premier ordre [Sollen]. Peu importe ici que cette substitution s'effectue à l'issue d'un soulèvement violent contre les individus qui étaient jusqu'alors compétents, "légitimes", pour créer et amender l'ordre juridique ${ }^{142}[$ Sein].»

Il y a ainsi une nouvelle légitimité juridico-institutionnelle qui conduit à reconsidérer l'appréciation de validité des ordres juridiques ancien et nouveau.

Kelsen essaye toutefois de justifier sa position en affirmant que «ce n'est pas le principe de légitimité mais la révolution qui dépouille de leur validité les normes de l'ordre ancien. Elles perdent leur validité non seulement de facto mais aussi de jure ». Or, si l'on peut concevoir que les normes perdent leur validité de facto du fait de la révolution, seul le changement de légitimité juridico-institutionnel permet de comprendre

141. Hans Kelsen, TGDE, op. cit., p. 171-172.

142. Ibid., p. 171. 
cette extension à la validité de jure. Arrivé à ce point de sa démonstration, ne parvenant plus à rester dans le domaine purement normatif, Kelsen est alors contraint de recourir au "principe d'efficacité » (fait), révélant l'intrication profonde entre l'être et le devoir-être, d'une part, et la complexité d'une validité plurielle (validité formelle, effectivité, légitimité), d'autre part: «si l'ancien ordre cesse d'être efficace et que l'ordre nouveau le devienne - parce que les individus dont la conduite est réglée par l'ordre nouveau se comportent effectivement, dans l'ensemble, en accord avec l'ordre nouveau -, alors cet ordre sera considéré comme un ordre juridique valide ${ }^{143}$.»

Deux constats doivent alors être opérés :

- Le jusnormativiste devrait apprécier, tel un «sociologue» (qu'il ne peut pourtant être selon Kelsen ${ }^{144}$ ), l'adéquation du comportement global effectif des individus à l'ordre juridique nouveau. Kelsen nous encourage donc à braver l'interdit majeur que lui-même a érigé: le syncrétisme des méthodes (normatives et causales).

- C'est parce que, à la suite de cette interprétation sociologique, l'ordre juridique nouveau est considéré comme efficace, qu'il peut, subséquemment, être interprété comme valide.

Pourtant, Kelsen se déjuge quelques lignes après en soutenant que les «normes sont valides parce qu'elles sont créées selon un procédé constitutionnel, et non parce que l'ordre juridique total est efficace ${ }^{145}$ ».

La distinction entre condition per quam (procédure de création conforme ou légalité formelle) et condition sine qua non (efficacité) apparaît alors réellement peu satisfaisante. Et s'il s'agit de deux conditions, on ne voit en outre pas pourquoi l'une est qualifiée de validité, tandis que l'autre est qualifiée de "principe d'efficacité ", ni pourquoi la validité devrait être totalement distincte de l'efficacité puisqu'il existe manifestement un rapport d'interdépendance. Si l'on ajoute le «principe

143. Ibid., p. 172.

144. Kelsen reconnaît bien comme scientifique l'activité du sociologue du droit, et il prescrit même à celle-ci d'être différente de celle du «simple» sociologue. Mais tandis que la science normative du droit étudie les normes et les comportements définis par celles-ci, le sociologue étudie les comportements effectifs. C'est pourquoi, bien que «la sociologie présuppose le concept juridique de droit, concept défini par la science normative du droit ", Kelsen estime que les deux sciences du droit sont apparentées mais non identiques (ibid., p. 230-231). «La sociologie du droit présuppose ce concept. Elle ne prend pas pour objet les normes valides - objet de la science normative du droit - mais la conduite humaine» (ibid., p. 227).

145. Ibid., p. 173. 
de légitimité » qui, bien que censé, selon Kelsen, n'être «plus valide dans le cas d'une révolution", permet toutefois à l'Autrichien de qualifier l'ordre juridique nouveau d'illégitime au regard des règles de l'ancien, et de légitime sur le plan institutionnel (ce qui le conduit au final à apprécier une légitimité à la fois factuelle et normative), force est de constater que le concept de légitimité a pour fonction principale de permettre la préservation de la distinction être/devoir-être. D'ailleurs cette dichotomie est maintenue, mais au prix de contorsions scientifiques (pour ne pas dire d'une certaine confusion). Ainsi, alors qu'il évoque en premier lieu l'efficacité de l'ordre juridique nouveau envisagé globalement, Kelsen opère ensuite une transition logico-sémantique et évoque celle des normes, ce qui lui permet d'affirmer que l'efficacité n'est pas une condition per quam de la validité - ce qu'il venait pourtant d'énoncer. N'étant plus une condition "réelle», l'efficacité, qui appartient au monde de l'être, peut alors être exclue d'une validité dont le caractère unidimensionnel (formel) nous apparaît désormais bel et bien artificiel ${ }^{146}$.

Servant de pont entre le monde de l'être et celui du devoir-être, le principe de légitimité n'est pourtant jamais considéré par Kelsen

146. Otto Pfersmann l'admet d'ailleurs lorsqu'il se prononce, en 1995, "Pour une typologie modale de classes de validité normative» (Otto Pfersmann, "Pour une typologie des classes de validité normative», dans Jean-Luc Petit (dir.), La Querelle des normes, Cahiers de philosophie politique et juridique, $\mathrm{n}^{\circ} 27$, Caen, Presses universitaires de Caen, 1995, en particulier p. 88-89): adoptant une perspective " ouvertement constructiviste » (p. 74), Otto Pfersmann relève que "La distinction entre "raison" [soit l'idée d'inférence] et "condition" [soit l'efficacité] avait été introduite pour maintenir une stricte séparation entre l'être et le devoir-être qui semblait être remise en question par la liaison entre un moment normatif et un moment factuel. Mais la différence de qualité entre ces deux conditions ne peut être marquée par une différence purement verbale qui ne modifie en rien leur commune propriété d'être des conditions [cumulatives]» (p. 89). Il en tire même la seule conclusion possible: «En dernier lieu la validité se confond avec l'application factuelle: la norme du degré le plus élevé est valide, si l'organe qui est responsable de son application l'applique effectivement. Mais que dire avant qu'il n'ait agit ainsi ? Ce serait alors au théoricien de justifier pourquoi la "norme" est valide ou pourquoi elle ne l'est pas» (p. 91). Mais, de cette conclusion qui donne pourtant à voir comment le normativisme fait effectivement du scientifique et du praticien «des décideurs » "à la place de l'organe juridique» (p. 91), Otto Pfersmman refuse de tirer les conséquences et propose, paradoxalement, un retour à la « conception restrictive » (p. 88) de Kelsen: « il faut alors supposer valides les normes régulièrement édictées dans un système grosso modo efficace» (p. 91). Il finit même par rejeter tout lien entre légalité formelle et efficacité en soulignant que «L'efficacité sera donc considérée comme une donnée indépendante» (p. 93). Et «il faut renoncer à la CVE [double condition cumulative de validité et d'efficacité] pour les normes, prises singulièrement» (p. 91). 
comme une composante de la validité d'un ordre juridique. Le principe kelsénien de «légitimité » a souvent été perçu, à tort, comme un simple équivalent de la légalité formelle. Résumant la place accordée à la légitimité au sein de la communauté des juristes, Jean-Marc Février énonce: "Ainsi, de façon générale, est légitime toute autorité ou tout acte établi conformément au droit positif. [Et l'auteur de citer le principe de légitimité de Kelsen au sens défini ici comme premier, soit comme condition de la validité ${ }^{147}$, et d'en conclure:] La légitimité serait donc pour le juriste une notion superfétatoire, une autre manière de désigner la validité ${ }^{148}$.»

Or, nous avons vu, d’une part, que la relation entre «légitimité » et "validité » n'était pas une relation d'identité, mais une relation causale et, d'autre part, que le construit kelsénien relatif audit principe, bien que confus, était bien plus élaboré, qu'il impliquait notamment une relation d'interdépendance entre les concepts de légitimité, de légalité formelle et d'effectivité. Pourtant, dernière marque de l'inachèvement du constructivisme kelsénien, seule la légalité formelle est considéré comme «validité».

Il nous faut maintenant tirer toutes les conclusions des constats opérés précédemment.

\section{Conclusions analytiques}

- le (néo)normativisme est un constructivisme;

- le (néo)normativisme est un constructivisme de projet;

- c'est parce que le projet (néo)normativiste est réducteur que le construit qui en découle l'est également;

- c'est le projet kelsénien qui fait de la science du droit une activité prescriptive;

- tout système juridique marqué par la présence d'une entité doctrinale forte est un système externe: l'unité de structure dudit système étant un terminus ad quem de la doctrine ${ }^{149}$;

147. «Le principe qu'une norme ne peut être posée que par l'organe compétent, c'està-dire par l'organe habilité à cet effet par une norme supérieure, est le principe de légitimité » (Hans Kelsen, TPD, op. cit., p. 272, cité par Jean-Marc Février, "Sur l'idée de légitimité », Revue de la Recherche juridique. Droit prospectif, 2002-1, p. 367-379, en particulier p. 367).

148. Jean-Marc Février, ibid., p. 367.

149. La distinction entre système interne (ou le système est considéré comme déjà organisé) et système externe revient à Mario Losano (Sistema e struttura nel diritto, Turin, G. Giapichelli, 1968, cité par Michel Troper, Pour une théorie juridique de l'État, op. cit., p. 161). 